\title{
New Innovation of low cost solar still
}

\author{
${ }^{1}$ S H Sengar, ${ }^{1} Y$ P Khandetod, ${ }^{1}$ A G Mohod
}

\begin{abstract}
Different solar stills were developed and evaluated for comparison with other solar stills available in market. Comparatively more distilled water was obtained from the solar still having an area of $1 \mathrm{~m}^{2}$ fabricated in fiber or metal body with glass glazing like single slope ,double slope and wick type solar still. Average maximum temperature and humidity was more in double slope, single slope and wick type solar still and hence the average quantity of distilled water obtained as $1350 \mathrm{ml} /$ day, 1550 $\mathrm{ml} /$ day and $2450 \mathrm{ml} /$ day respectively. The plastic made up of $W$-shape solar still provided with 3 channels produce maximum distilled water as $2104 \mathrm{ml} /$ day where as $W$ shape solar still with two channels erected on concrete and ground produced only $1012 \mathrm{ml} /$ day and $1443 \mathrm{ml} /$ day respectively. L shape solar still produce average distilled water was $925 \mathrm{ml} /$ day which was very low among the all type of solar still. Comparative cost of compact nature of solar stills like single slope, double slope and wick type solar still was more than Rs.7000/- which is four times more than newly developed $W$-shape 3 channel solar still. Comparative output from newly developed solar still was low but it has several advantages that it is cheapest, cost efficient and easy to clean. Concentration of $\mathrm{pH}, \mathrm{EC}$, TDS and ions in solar distilled water was found to be similar as conventional distilled water. The cost of $\mathrm{W}$ shape three channel solar still is recovered within 4 months 6 days only.
\end{abstract}

Keywords: solar stills, chemical analysis, economics

${ }^{1}$ Department of Electrical and Other Energy Sources, College of Agricultural Engineering and Technology, DBSKKV, Dapoli, India 


\section{Methodology}

Wick type, W shape and L shape of solar stills were designed on the basis of solar declination angle, slope of collector and available insolation. During theoretical design calculations of solar still (Plate 1), peak winter season was considered. In winter season, December month was selected for finding the solar declination angle $(\delta)$, Slope of collector $(\beta)$, intensity of insolation on horizontal and vertical surface and value of $\operatorname{Cos} \theta$ is shown in Table 1. The newly developed solar stills were evaluated for load test and compared with the output of single and double slope solar still available in market.

Table: 1. Details of design calculations for even type solar still.

\begin{tabular}{|c|c|c|c|}
\hline $\begin{array}{l}\text { S. } \\
\text { N. }\end{array}$ & Particulars & Symbol & Design parameter of solar still \\
\hline 1 & Solar declination angle & $\delta$ & $\begin{array}{l}\delta=23.45 \sin [0.9863(284+\mathrm{n})] \\
\delta=-23.3\end{array}$ \\
\hline 2 & Slope of collector & $\beta$ & $\begin{array}{l}\beta=(\Phi-\delta) \\
\beta=40^{\circ} 48^{\prime}\end{array}$ \\
\hline 3 & $\begin{array}{l}\text { Intensity of insolation on } \\
\text { horizontal }\end{array}$ & $I_{c}$ & $\begin{array}{l}\mathrm{I}_{\mathrm{c}}=\mathrm{I}_{\mathrm{h}} \times \operatorname{Cos} \theta \\
\mathrm{I}_{\mathrm{c}}=450 \mathrm{~W} / \mathrm{m}^{2}\end{array}$ \\
\hline 4 & $\begin{array}{l}\text { Intensity of insolation on } \\
\text { sloping surface }\end{array}$ & $I_{s}$ & $\begin{array}{l}\mathrm{I}_{\mathrm{s}}=\mathrm{I}_{\mathrm{h}} \times \operatorname{Cos} \theta / \operatorname{Cos} \theta_{\mathrm{h}} \\
\mathrm{I}_{\mathrm{s}}=594.5 \mathrm{~W} / \mathrm{m}^{2}\end{array}$ \\
\hline 5 & Cosine of $\theta_{\mathrm{h}}$ & $\theta_{\mathrm{h}}$ & $\theta_{\mathrm{h}}=40^{\circ} .8^{\prime}$ \\
\hline
\end{tabular}

\section{Wick Type Solar still}

Wick Type Solar still was fabricated at the central workshop of the College of agricultural Engineering and Technology, Dapoli. The pictorials view with different component of wick type solar still is shown in Plate1.

The device was consisted of a base frame made up of angle irons of size $25 \mathrm{~mm} \times 25 \mathrm{~mm} \times 4 \mathrm{~mm}$, which was $1 \mathrm{~m} \times 1 \mathrm{~m}$ in size and formed the rigid base for the complete unit.

The main frame was also made up of angle irons of size $25 \mathrm{~mm} \times 25 \mathrm{~mm}$ x $3 \mathrm{~mm}$, rested on the base frame and hinged from one side for the angle adjustment. The frame was enclosed with the 18 gauge and 24 gauge GI sheets and a thermacol was placed in between the two sheets as insulation from all sides 
except from top. The absorber consisted of a corrugated sheet of 24 gauge and of $1 \mathrm{~m}^{2}$ area for increasing absorption of incident solar radiations. The unit was painted with blackboard paint for attaining higher absorption of incident solar radiations. The unit was covered with a glass cover of $5 \mathrm{~mm}$ thick plane glass, fitted in aluminum frame having an area of $1 \mathrm{~m}^{2}$. The greenhouse effect was possible due to glass which entrapped long wave radiations. The collected water vapour got condensed on inner side of glass.

Device was basically divided into three components as heating chamber, cooling cover and collection unit. Inside the heating chamber, GI pipe was provided for continuous water supply with 15 pin holes of $3 \mathrm{~mm}$ drill. The pipe was connected to a storage tank for continuous water supply.

The jute was used as a wick material, which was laid on the corrugated absorber plate with sufficient open area for exposing the black ridge of corrugated absorber. The wick material absorbed the droplets of water coming out from GI pipe and carried this water along the length of the material by capillary action. During this process unused water was collected through the drain outlet and distilled water was collected separately from opposite side and collected in beaker.

The water which was evaporated at higher temperature inside the heating chamber got condensed on the glass surface. The channel was provided for its collection. Water vapour inside the heating chamber got condensed in small droplets of liquid due to low temperature of glass cover. The total cost of this solar still was Rs.7241/- shown in Table 2.

\section{Low cost ( W-shape) solar still}

Low cost (W-shape) solar still frame was design and fabricated at the central workshop of the College of Agricultural Engineering and Technology (CAET), Dapoli. This still was fabricated using $25 \mathrm{~mm}$ diameter M.S. pipe to make the frame as shown in Plate 2. The pipes and corners were well polished and laminated in order to avoid corrosions as well as to protect the polythene sheet from damage. The size of basin was $2 \mathrm{~m}$ x $2 \mathrm{~m}$ was prepared with black plastic covered basin in soil (Plate-2), in which water was impounded. The UV stabilized 200 micron polythene sheet of size 4 x $2.5 \mathrm{~m}$ was wrapped properly over the frame so that it became leak proof. The distilled water-collecting channel made from GI sheet wrapped with plastic was attached below to the frame with the help of non-corrosive wires. 
Table: 2. Materials used in wick type solar still

\begin{tabular}{|c|c|c|c|c|c|}
\hline $\begin{array}{c}\text { Sr.N } \\
\text { o. }\end{array}$ & Item & Specifications & $\begin{array}{l}\text { Weight } \\
\text { (kg) }\end{array}$ & $\begin{array}{l}\text { Rate } \\
\text { (Rs.) }\end{array}$ & $\begin{array}{l}\text { Cost } \\
\text { (Rs.) }\end{array}$ \\
\hline 1 & M.S. Angle & $25 \mathrm{~mm} \times 25 \mathrm{~mm} \times 4 \mathrm{~mm}$ & 9 & 60 & $540 /-$ \\
\hline 2 & M.S. Angle & $25 \mathrm{~mm} \times 25 \mathrm{~mm} \times 3 \mathrm{~mm}$ & 12 & 60 & $720 /-$ \\
\hline 3 & GI Sheet & 18 gauge & 21 & 75 & $1575 /-$ \\
\hline 4 & GI sheet & 24 gauge & 2 & 75 & $150 /-$ \\
\hline 5 & Corrugated sheet & 24 gauge & 2 & 150 & $400 /-$ \\
\hline 6 & M.S. Flat & $25 \mathrm{~mm} \times 4 \mathrm{~mm}$ & 3.5 & 45 & $158 /-$ \\
\hline 7 & GI pipe & $63 \mathrm{~mm}$ diameter & $2 \mathrm{~m}$ & $200 / \mathrm{m}$ & $400 /-$ \\
\hline 8 & M.S. rod & $5 \mathrm{~mm}$ diameter & 4 & 45 & $180 /-$ \\
\hline 9 & Bucket plastic pipe & & & & $100 /-$ \\
\hline 10 & Thermocol & $25 \mathrm{~mm}$ thick & & $\begin{array}{l}30 / \text { She } \\
\text { et }\end{array}$ & $120 /-$ \\
\hline 11 & Hinges (2) & $25 \mathrm{~mm} \times 25 \mathrm{~mm}$ & & $\begin{array}{l}30 / \mathrm{Pie} \\
\mathrm{ce}\end{array}$ & $60 /-$ \\
\hline 12 & Lambi & & $100 \mathrm{gm}$ & & $50 /-$ \\
\hline 13 & Red oxide & & $100 \mathrm{gm}$ & & $30 /-$ \\
\hline 14 & Blackboard paint & & $500 \mathrm{ml}$ & & $50 /-$ \\
\hline 15 & Gromate & & & $\begin{array}{l}\text { 5/Piec } \\
\mathrm{e}\end{array}$ & $10 /-$ \\
\hline 16 & Cock (Plastic) & & & $\begin{array}{l}\text { 5/Piec } \\
\mathrm{e}\end{array}$ & $5 /-$ \\
\hline 17 & Araldite (2) & & & $\begin{array}{l}30 / \mathrm{Pie} \\
\text { ce }\end{array}$ & $60 /-$ \\
\hline 18 & Glass with frame & $1 \mathrm{~m} \times 1 \mathrm{~m}$ & & & $942 /-$ \\
\hline 19 & Nut and Bolts (10) & & & & $20 /-$ \\
\hline \multirow[t]{2}{*}{20} & Labour Charges & $30 \%$ & & & 1671 \\
\hline & & Total & & & 7241/- \\
\hline
\end{tabular}

The collecting channels were fabricated so as to catch the condensed droplets of water inside the solar still. The material used for the fabrication of this unit is presented in Table 3. This ( $W$-shape) solar still frame along with UV stabilized plastic as glazing kept over the basin dug on ground (Plate 2) and making it leak 
proof by using soil cover. The water present in basin got evaporated due to higher temperature inside the heating chamber. Water vapour inside the heating chamber got condensed in the form of small droplets of water due to lower temperature on inner side of polythene. Condensed droplets of evaporated water were collected through three channels. Surrounding condensed water was collected through third channel from all sides at bottom (Plate 3). First two channels which fixed inside solar still (Plate 4)

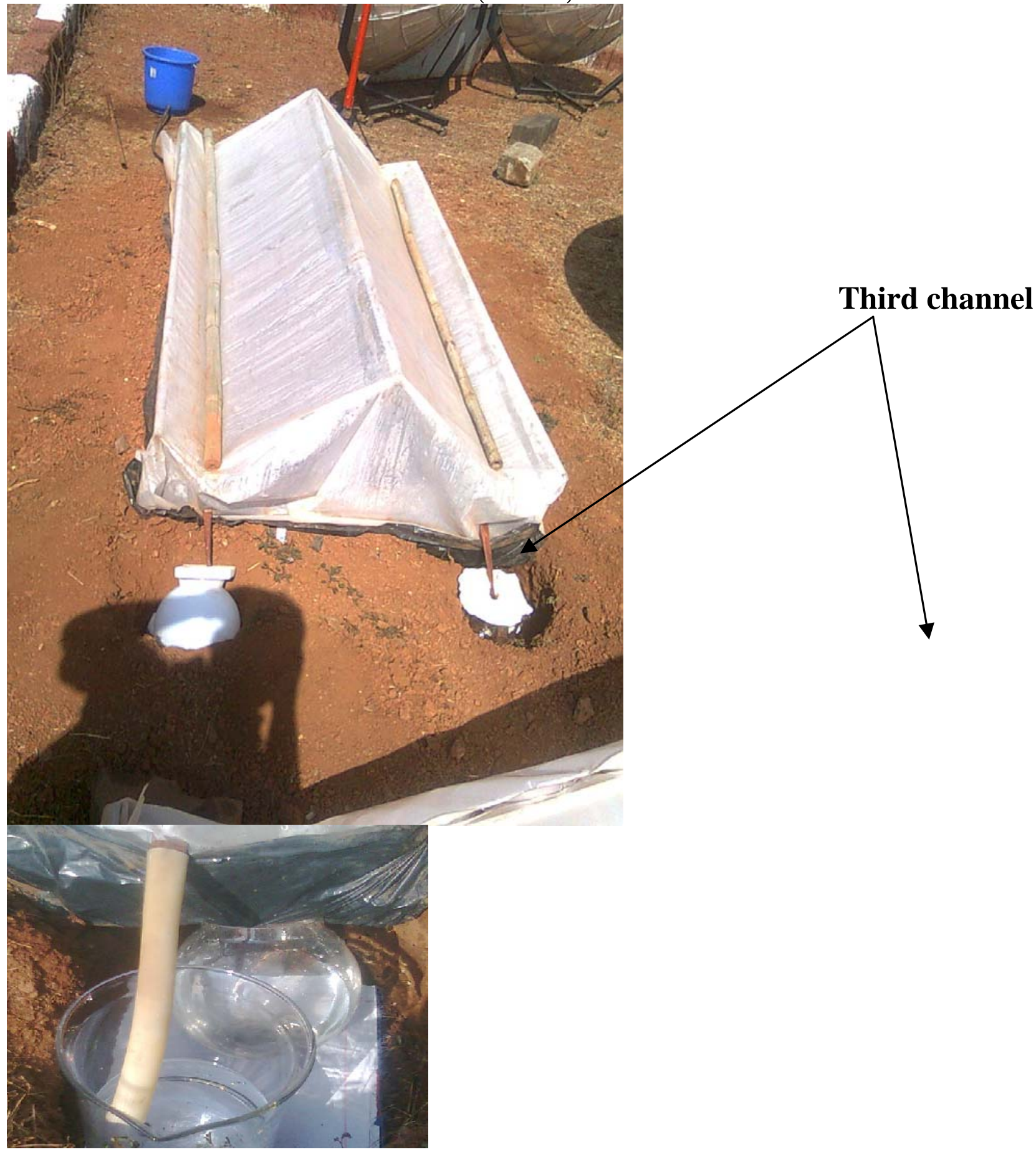

Plate: 4. Two channel provision for W-shape solar still Plate-3 Third channel 
W shape two channels solar still was also erected over the cement block (Plate 5) for performance of distilled water. The cost required to erect w shape solar still on ground and cement were Rs. 1848/- and 4000/-

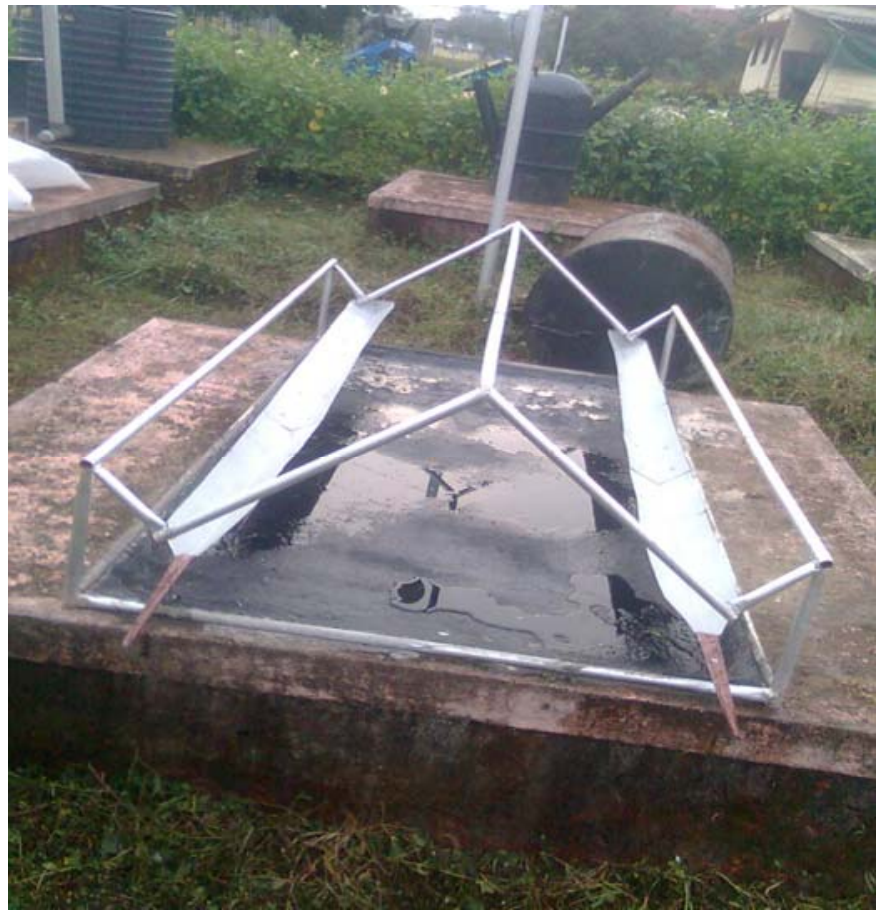

(a)

Plate: 5. W-shape solar still on concrete with two channel Low cost (L-shape) solar still

By using wooden strips and aluminum channel $\mathrm{L}$ shape solar still was developed with plastic glazing on soil for distilled water output through single channel. The size of $\mathrm{L}$ shape solar still was $2 \mathrm{~m}^{2}(1.4 \mathrm{~m} \times 1.4 \mathrm{~m})$. The total cost required to erect L shape solar still on ground was Rs.1000/- 


\begin{tabular}{|c|c|c|c|c|c|}
\hline $\begin{array}{l}\text { Sr. } \\
\text { No. }\end{array}$ & Item & Specification & $\begin{array}{l}\text { Quantity } \\
\text { required }\end{array}$ & $\begin{array}{c}\text { Rate of } \\
\text { item }\end{array}$ & $\begin{array}{l}\text { Total } \\
\text { Cost } \\
\text { (Rs.) }\end{array}$ \\
\hline 1 & M. S. pipe(Low grade) & $\begin{array}{l}25 \mathrm{~mm} \\
\text { diameter }\end{array}$ & $15.8 \mathrm{~m}$ & $40 / \mathrm{m}$ & $732 /-$ \\
\hline 2 & Polythene film & & $4 \times 2.5 \mathrm{~m}$ & $50 / \mathrm{m}^{2}$ & $500 /-$ \\
\hline 3 & GI sheet & 18 gauge & $0.3 \times 2.2 \mathrm{~m}$ & $220 / \mathrm{m}^{2}$ & $150 /-$ \\
\hline 4 & Red paint & & $100 \mathrm{gm}$ & $600 / \mathrm{kg}$ & $60 /-$ \\
\hline 5 & Black paint & & $500 \mathrm{gm}$ & $240 / \mathrm{kg}$ & $120 /-$ \\
\hline 6 & Cement & 53 grade & $5 \mathrm{~kg}$ & $10 / \mathrm{kg}$ & $50 /-$ \\
\hline 7 & Sand & Fine & $10 \mathrm{~kg}$ & & $40 /-$ \\
\hline 8 & Welding rod & Short length & 8 rods & $12 / \mathrm{rod}$ & $96 /-$ \\
\hline 9 & Labor charge & & 2 days & Rs.100/day & 200 \\
\hline
\end{tabular}

TOTAL

$1848 /-$

\section{Results and Discussions \\ Performance Evaluation}

Developed solar stills were evaluated for winter and summer months with load test at $40^{\circ}$ angle. Devices were tested for comparison with the output of distilled water from single and double slope still units available in market.

\section{Wick type}

No load test

Wick type solar still having area $1 \mathrm{~m}^{2}(1 \mathrm{~m} \times 1 \mathrm{~m})$ is evaluated for no load test in winter. Maximum temperature was obtained at 1P.M.in solar still, where solar intensity was $503 \mathrm{~W} / \mathrm{m}^{2}$, inside temperature was $91^{\circ} \mathrm{C}$, ambient temperature was $31^{\circ} \mathrm{C}$ and outside relative humidity was 45.6 per cent. The trend obtained in no load test during performance of bare still unit is shown in Table 4. It was observed from Fig 1 that as inside temperature increased, inside relative humidity decreased and vice versa. It was also observed that the temperature inside the solar still increased with outside solar radiation. In no load test in summer, maximum temperature was obtained at 2 P.M., where solar intensity was 618 $\mathrm{W} / \mathrm{m}^{2}$, inside temperature was $94^{\circ} \mathrm{C}$, ambient temperature was $36.5^{\circ} \mathrm{C}$ and 
outside relative humidity was $34.5 \%$. The trend obtained in no load test during performance of bare solar still is shown in Table 5. It was observed (Fig 2) that as inside temperature increased, inside relative humidity decreased and vice versa. It was also observed that the temperature inside the solar still increased with outside solar radiation.

Table: 4. Performance of wick type solar still with no load test in winter

\begin{tabular}{cccccc}
\hline Time & Insolation & $\begin{array}{c}\text { Inside R.H. } \\
\mathbf{( \% )}\end{array}$ & $\begin{array}{c}\text { Inside } \\
\text { Temp. } \\
\mathbf{(} \mathbf{C})\end{array}$ & $\begin{array}{c}\text { Outside } \\
\text { Temp. } \\
\left.\mathbf{(}{ }^{\circ} \mathbf{C}\right)\end{array}$ & $\begin{array}{c}\text { Outside R.H. } \\
\mathbf{( \% )}\end{array}$ \\
\hline 8.00 & 98 & 88.6 & 22 & 18 & 89.5 \\
9.00 & 156 & 78.5 & 46 & 24 & 78.3 \\
10.00 & 212 & 65.4 & 59 & 27 & 65.4 \\
11.00 & 423 & 58.5 & 65 & 28 & 52.3 \\
12.00 & 446 & 52.1 & 79 & 30 & 44.8 \\
13.00 & 503 & 46.9 & 91 & 31 & 45.6 \\
14.00 & 444 & 37.6 & 89 & 31 & 44.7 \\
15.00 & 398 & 38.5 & 78 & 28 & 53.1 \\
16.00 & 234 & 45.9 & 70 & 27 & 56.9 \\
17.00 & 120 & 53.6 & 64 & 27 & 67.8 \\
18.00 & 37 & 61.7 & 62 & 24 & 78 \\
\hline
\end{tabular}

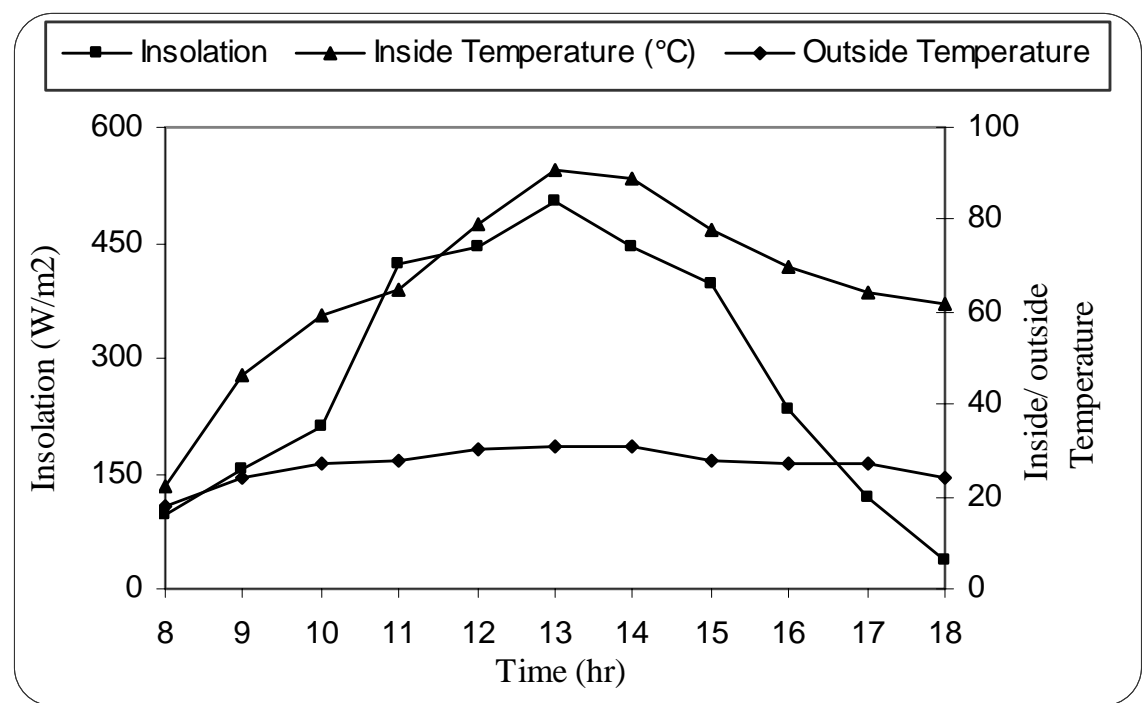

Fig. 1. Performance of wick type solar still no load test in winter 
Table: 5. Performance of wick type solar still with no load test in summer

\begin{tabular}{|c|c|c|c|c|c|}
\hline $\begin{array}{l}\text { Time } \\
\text { (Hrs) }\end{array}$ & $\begin{array}{l}\text { Insolation } \\
\left(\mathrm{W} / \mathrm{m}^{2}\right)\end{array}$ & $\begin{array}{c}\text { Inside } \\
\text { R.H. } \\
(\%)\end{array}$ & $\begin{array}{c}\text { Inside Temp. } \\
\left({ }^{\circ} \mathrm{C}\right)\end{array}$ & $\begin{array}{l}\text { Outside } \\
\text { Temp. } \\
\left({ }^{\circ} \mathrm{C}\right)\end{array}$ & $\begin{array}{c}\text { Outside } \\
\text { R.H. } \\
(\%)\end{array}$ \\
\hline 8.00 & 233 & 89.7 & 35.5 & 23.4 & 79.1 \\
\hline 9.00 & 343 & 71.1 & 47.1 & 26.9 & 66.5 \\
\hline 10.00 & 453 & 56.4 & 57.0 & 29.1 & 50.3 \\
\hline 11.00 & 546 & 48.2 & 65.2 & 31.1 & 46.5 \\
\hline 12.00 & 555 & 39.5 & 76.5 & 34.2 & 40.9 \\
\hline 13.00 & 598 & 36.1 & 87.3 & 36.0 & 36.7 \\
\hline 14.00 & 618 & 36.8 & 94.0 & 36.5 & 34.5 \\
\hline 15.00 & 603 & 36.9 & 91.4 & 36.4 & 35.4 \\
\hline 16.00 & 489 & 49.0 & 83.5 & 32.7 & 48.9 \\
\hline 17.00 & 357 & 51.1 & 74.4 & 30.0 & 52.2 \\
\hline 18.00 & 232 & 56.3 & 66.8 & 28.5 & 59.8 \\
\hline
\end{tabular}

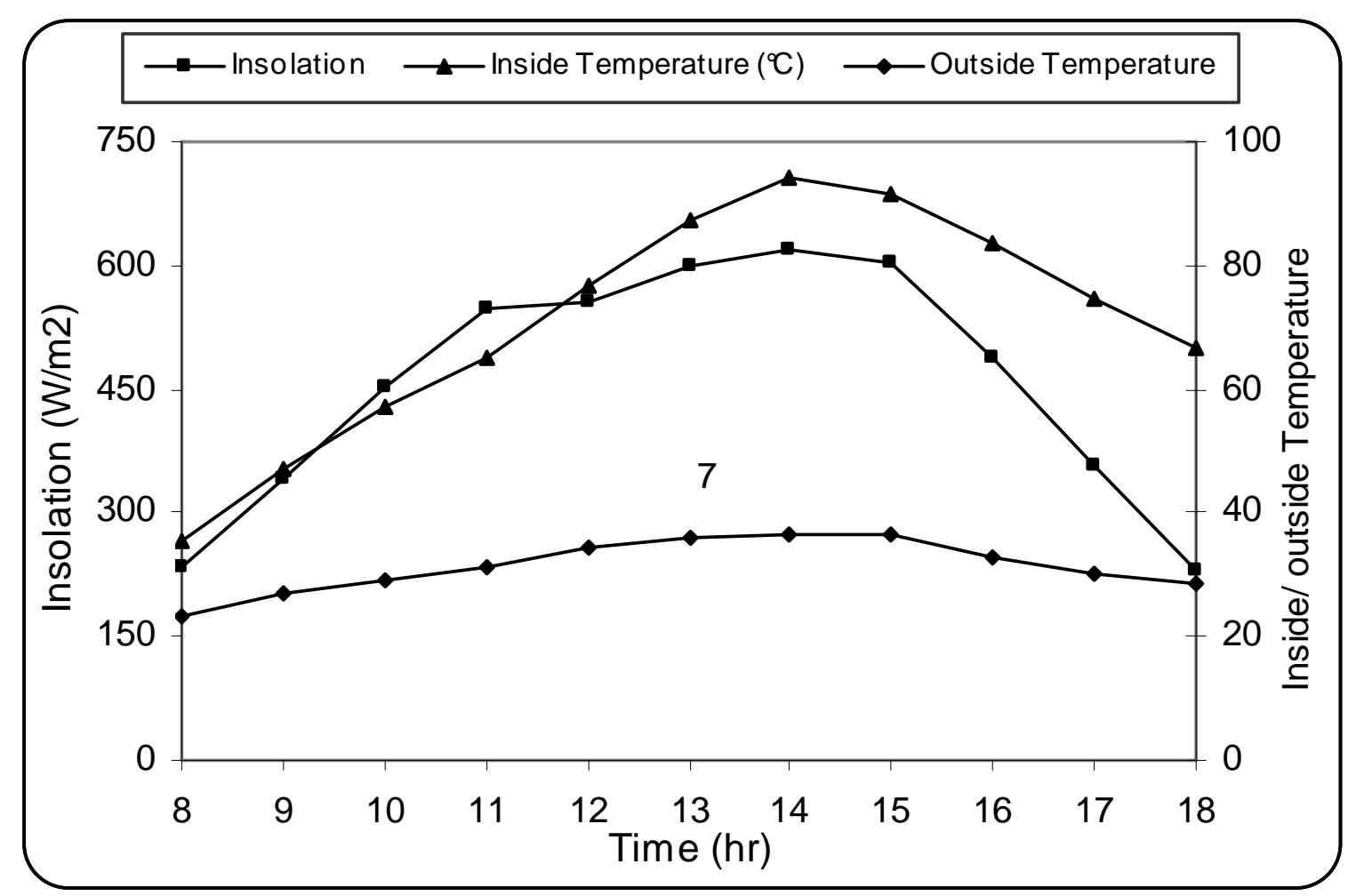

Fig. 2. Performance of wick type solar still no load test in summer Load test in winter

At $40^{\circ}$ angle of collector, maximum temperature obtained at 1 P.M was $81.0^{\circ} \mathrm{C}, \quad$ where solar intensity was $544 \mathrm{~W} / \mathrm{m}^{2}$ and ambient temperature was 
$32^{\circ} \mathrm{C}$ as shown in Table 6 . It was also observed that the maximum wind speed was $7.6 \mathrm{~m} / \mathrm{s}$ at 1P.M. and hence during that period the maximum distillation rate i.e. $300 \mathrm{ml} /$ hour was observed.

Table: 6. Performance of wick type solar still in winter

\begin{tabular}{|c|c|c|c|c|c|c|c|}
\hline $\begin{array}{l}\text { Time } \\
\text { (Hrs) }\end{array}$ & $\begin{array}{c}\text { Insola } \\
\text { tion }\end{array}$ & $\begin{array}{l}\text { Inside } \\
\text { R.H. } \\
(\%)\end{array}$ & $\begin{array}{c}\text { Inside } \\
\text { Temperat } \\
\text { ure }\left({ }^{\circ} \mathrm{C}\right)\end{array}$ & $\begin{array}{c}\text { Outside } \\
\text { Temperatu } \\
\text { re }\left({ }^{\circ} \mathrm{C}\right)\end{array}$ & $\begin{array}{c}\text { Outsid } \\
\text { e R.H. } \\
(\%)\end{array}$ & $\begin{array}{l}\text { Win } \\
\text { d } \\
\text { spee } \\
\text { d }\end{array}$ & $\begin{array}{c}\text { Cumulativ } \\
\text { e } \\
\text { Distillatio } \\
n(\mathrm{ml})\end{array}$ \\
\hline 8.00 & 98 & 71 & 34.8 & 21.3 & 88.5 & 0 & 0 \\
\hline 9.00 & 320 & 65.4 & 49.3 & 26.8 & 81.3 & 0 & 100 \\
\hline 10.00 & 443 & 69 & 65.3 & 27.3 & 67 & 2.1 & 290 \\
\hline 11.00 & 508 & 70.1 & 75.4 & 28.9 & 56.5 & 5.3 & 440 \\
\hline 12.00 & 550 & 73.4 & 77.6 & 31.6 & 44.1 & 6.9 & 650 \\
\hline 13.00 & 544 & 76.8 & 81 & 32 & 35.4 & 7.6 & 900 \\
\hline 14.00 & 520 & 100 & 77.4 & 33.5 & 37 & 2.9 & 1200 \\
\hline 15.00 & 453 & 100 & 64.1 & 35.1 & 38.5 & 2.5 & 1400 \\
\hline 16.00 & 323 & 92 & 62.3 & 36.8 & 46.9 & 2.5 & 1650 \\
\hline 17.00 & 61 & 91.6 & 36.7 & 30 & 58.6 & 0.5 & 1900 \\
\hline 18.00 & 36 & 98.4 & 35.9 & 28.4 & 72.4 & 0.2 & 2150 \\
\hline \multicolumn{7}{|c|}{ Overnight distillation up to 8.00 a.m. } & 150 \\
\hline \multicolumn{7}{|c|}{ Total } & 2300 \\
\hline
\end{tabular}

The trend obtained during performance showed in the Fig. 3 as in the beginning solar intensity increased, inside R.H. and temperature increased and once the R.H. attained $100 \%$ value, it remain unchanged invariant of solar intensity. Cumulative distilled water obtained from wick type solar still at $40^{\circ}$ in winter was $2300 \mathrm{ml}$ as shown in Table 6. 


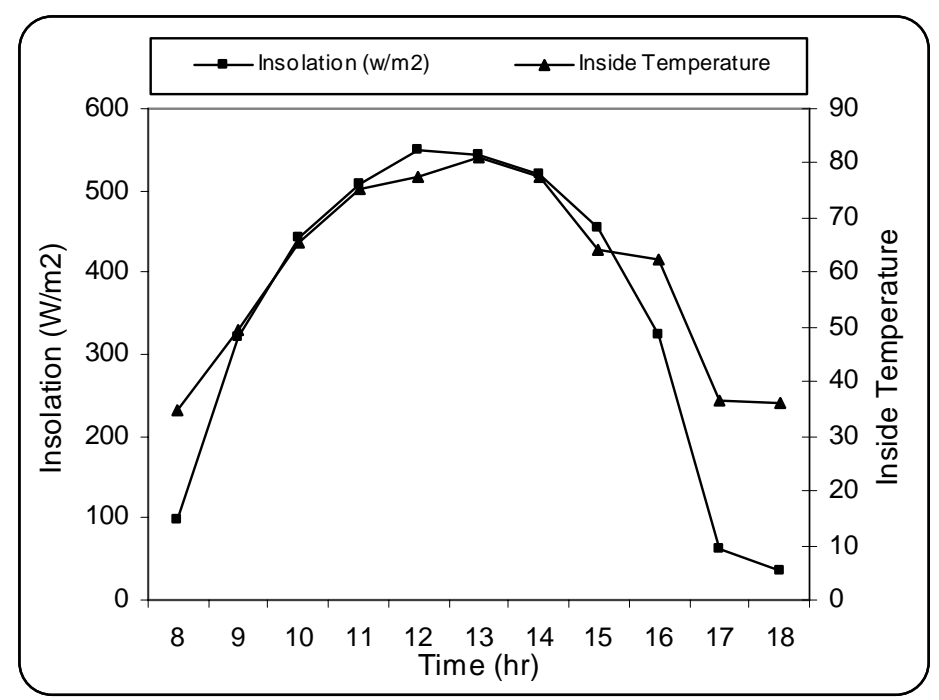

(a)

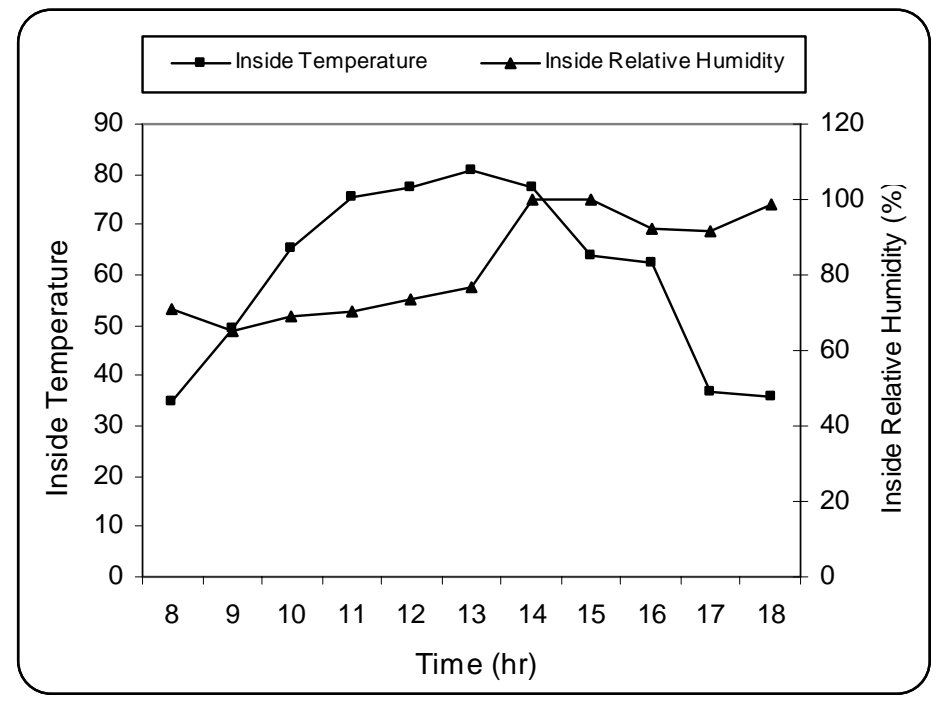

(b)

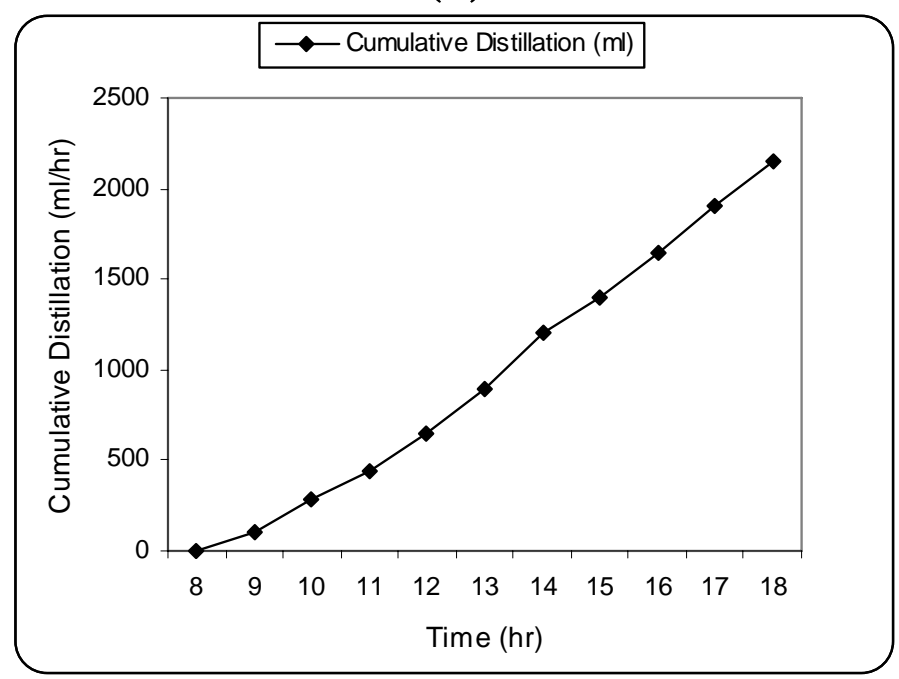

(c)

Fig. 3. Performance of wick type solar still with load in winter 


\section{Load test in summer}

During the performance of wick type solar still with wick material at $40^{\circ}$ angle of collector during summer, maximum temperature obtained at 1 P.M was $86.2^{\circ} \mathrm{C}$, when solar intensity was $600 \mathrm{~W} / \mathrm{m}^{2}$ and ambient temperature was $38.0^{\circ} \mathrm{C}$. The trend obtained as shown in Fig. 4 shows that distilled water obtained was minimum compared to winter. Maximum cumulative distilled water obtained was $2550 \mathrm{ml} /$ day.

Table: 7. Performance of wick type solar still in summer

\begin{tabular}{|c|c|c|c|c|c|c|c|}
\hline Time & $\begin{array}{l}\text { Insolation } \\
\left(\mathrm{W} / \mathrm{m}^{2}\right)\end{array}$ & $\begin{array}{c}\text { Inside } \\
\text { R.H. } \\
(\%)\end{array}$ & $\begin{array}{l}\text { Inside } \\
\text { Temp. } \\
\left({ }^{\circ} \mathrm{C}\right)\end{array}$ & $\begin{array}{l}\text { Outside } \\
\text { Temp. } \\
\left({ }^{\circ} \mathrm{C}\right)\end{array}$ & $\begin{array}{c}\text { Outsid } \\
\text { e R.H. } \\
\text { (\%) }\end{array}$ & $\begin{array}{l}\text { Wind } \\
\text { speed } \\
(\mathrm{m} / \mathrm{s} \\
)\end{array}$ & $\begin{array}{l}\text { Cumulative } \\
\text { Distillation } \\
\text { (ml) }\end{array}$ \\
\hline 8.00 & 234 & 72.3 & 45.2 & 27.1 & 72.1 & 0 & 0 \\
\hline 9.00 & 455 & 78.5 & 59.1 & 29.4 & 56.2 & 0.3 & 100 \\
\hline 10.00 & 549 & 76.4 & 76.4 & 30.8 & 41.1 & 5.1 & 300 \\
\hline 11.00 & 607 & 71.1 & 79.9 & 34.2 & 34.5 & 0.5 & 550 \\
\hline 12.00 & 617 & 69.4 & 81.5 & 36.7 & 32.1 & 2.2 & 800 \\
\hline 13.00 & 600 & 71.6 & 86.2 & 38 & 33.5 & 1.2 & 1000 \\
\hline 14.00 & 601 & 75.3 & 82.4 & 37.3 & 38.6 & 0.1 & 1250 \\
\hline 15.00 & 553 & 78.1 & 74.6 & 34.2 & 42.9 & 0 & 1500 \\
\hline 16.00 & 421 & 83.8 & 70.4 & 31.3 & 50.1 & 4.3 & 1800 \\
\hline 17.00 & 267 & 88.5 & 62.5 & 30.5 & 57 & 2.5 & 2100 \\
\hline 18.00 & 165 & 89 & 57.1 & 30.1 & 69.3 & 1.6 & 2350 \\
\hline \multicolumn{7}{|c|}{ Overnight distillation up to 8.00 a.m. } & 200 \\
\hline \multicolumn{7}{|c|}{ Total } & 2550 \\
\hline
\end{tabular}

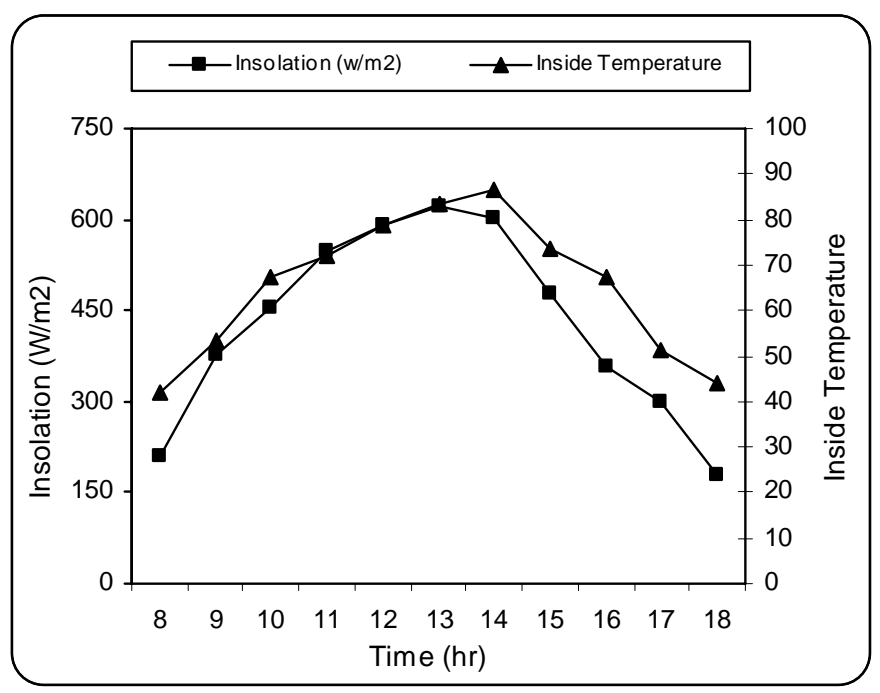


(a)

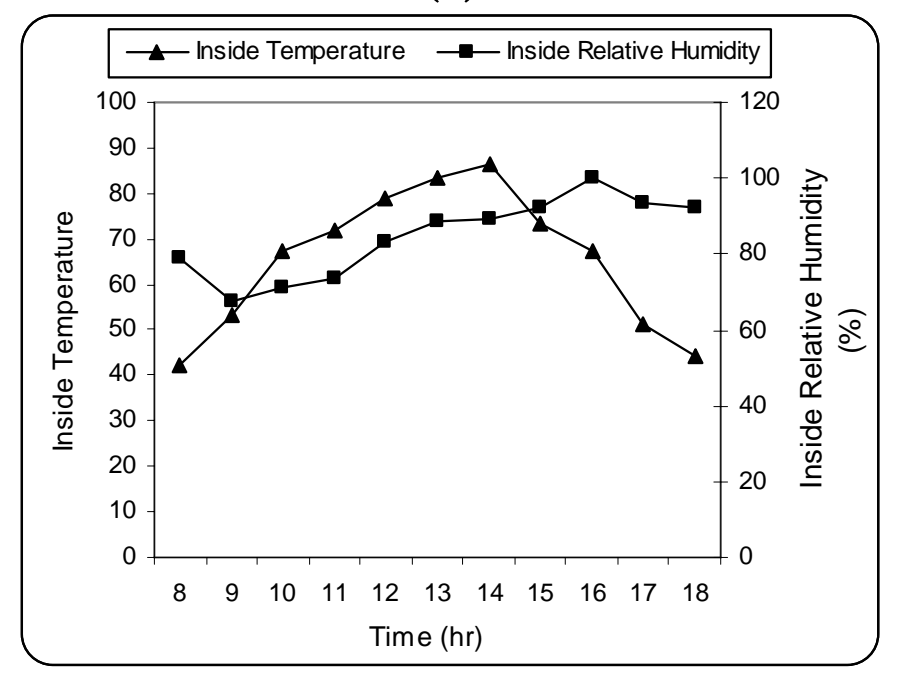

(b)

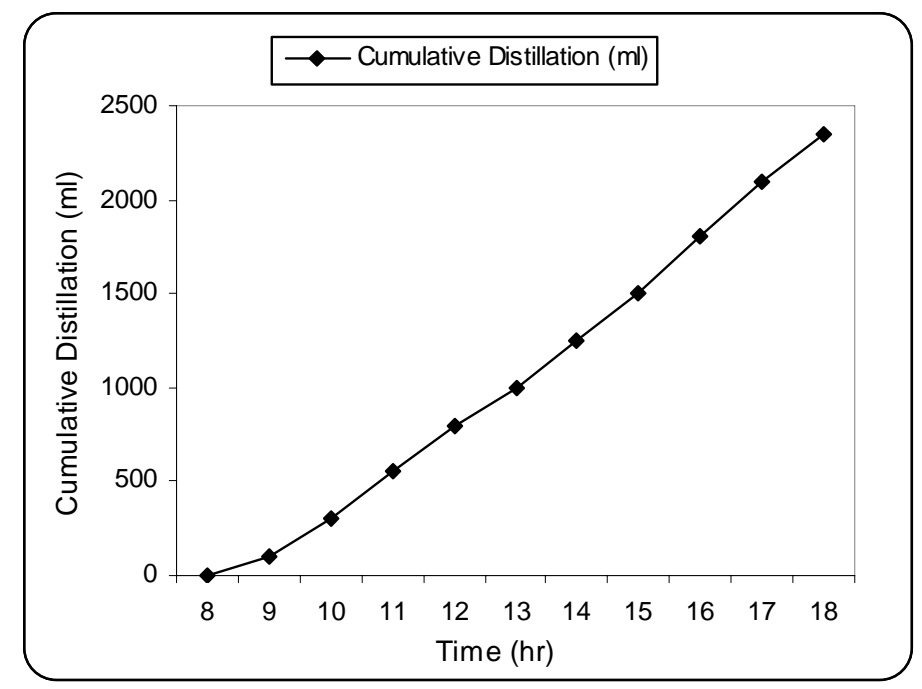

(c)

Fig. 4. Performance of wick type solar still with load in summer

\section{Selection of poly film materials}

Before using poly film as glazing material, it checked for its maximum transmitivity of solar radiation. Transitivity of polyfilm was checked out by using solarimter readings in $\mathrm{W} / \mathrm{m}^{2}$. The three type of polyfilm like plain polyfilm (silpolin), polyfilm antisulphur and UV stabilized polyfilm were selected for better transmitivity. The inside and outside solar radiations at same height were observed in clear sky condition for all selected polyfilm. It was observed that $76.83 \%$ solar radiation were transmitted from UV stabilized 200 micron white polyfilm where as $65.73 \%$ transmitivity observed in polyfim antisulphur and $69.97 \%$ in plain polyfil (silpolin) shown in Fig.5 ( a) and (b). 


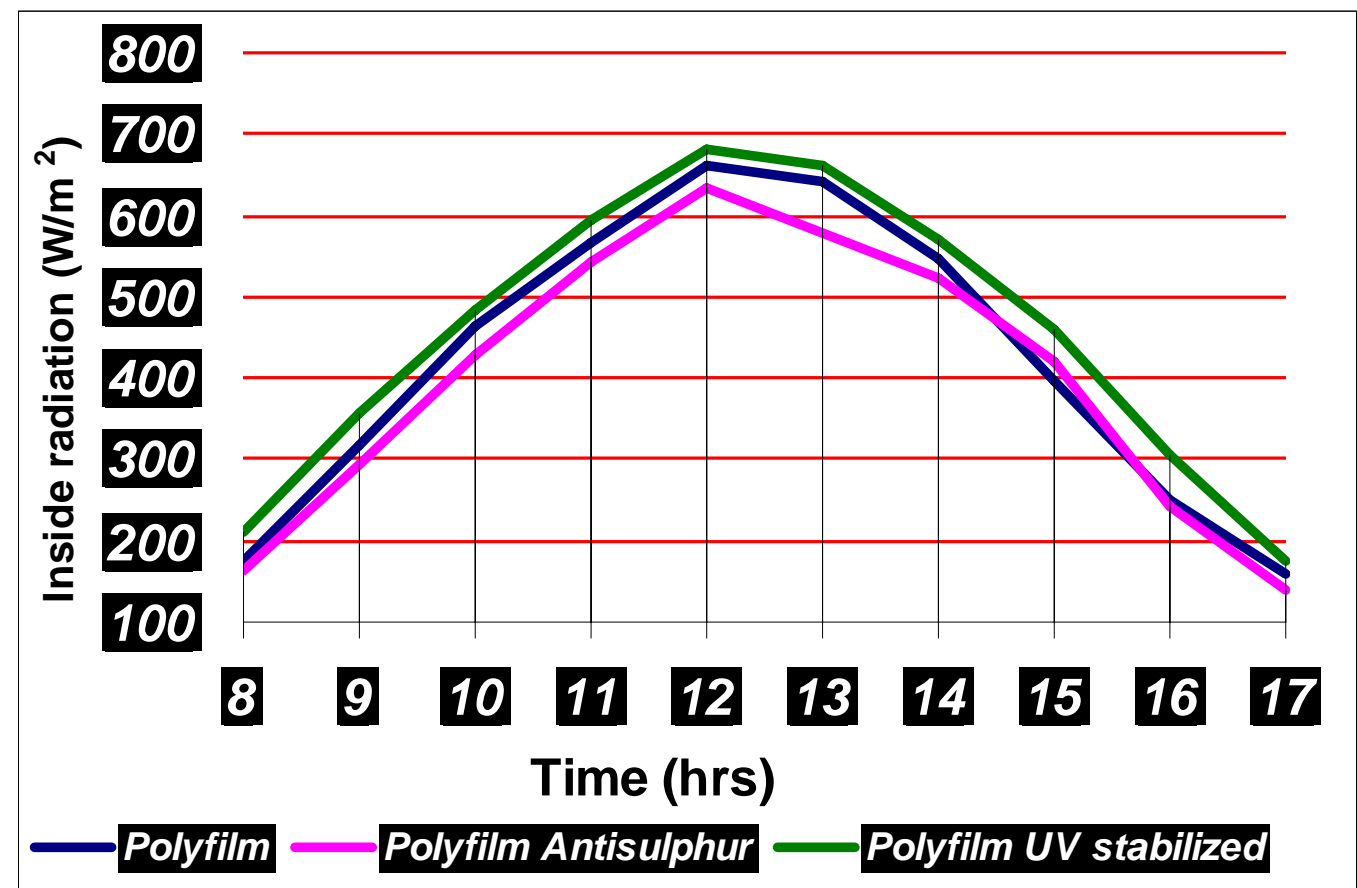

Fig.5. (a) Transmitivity of solar radiation inside poly film.

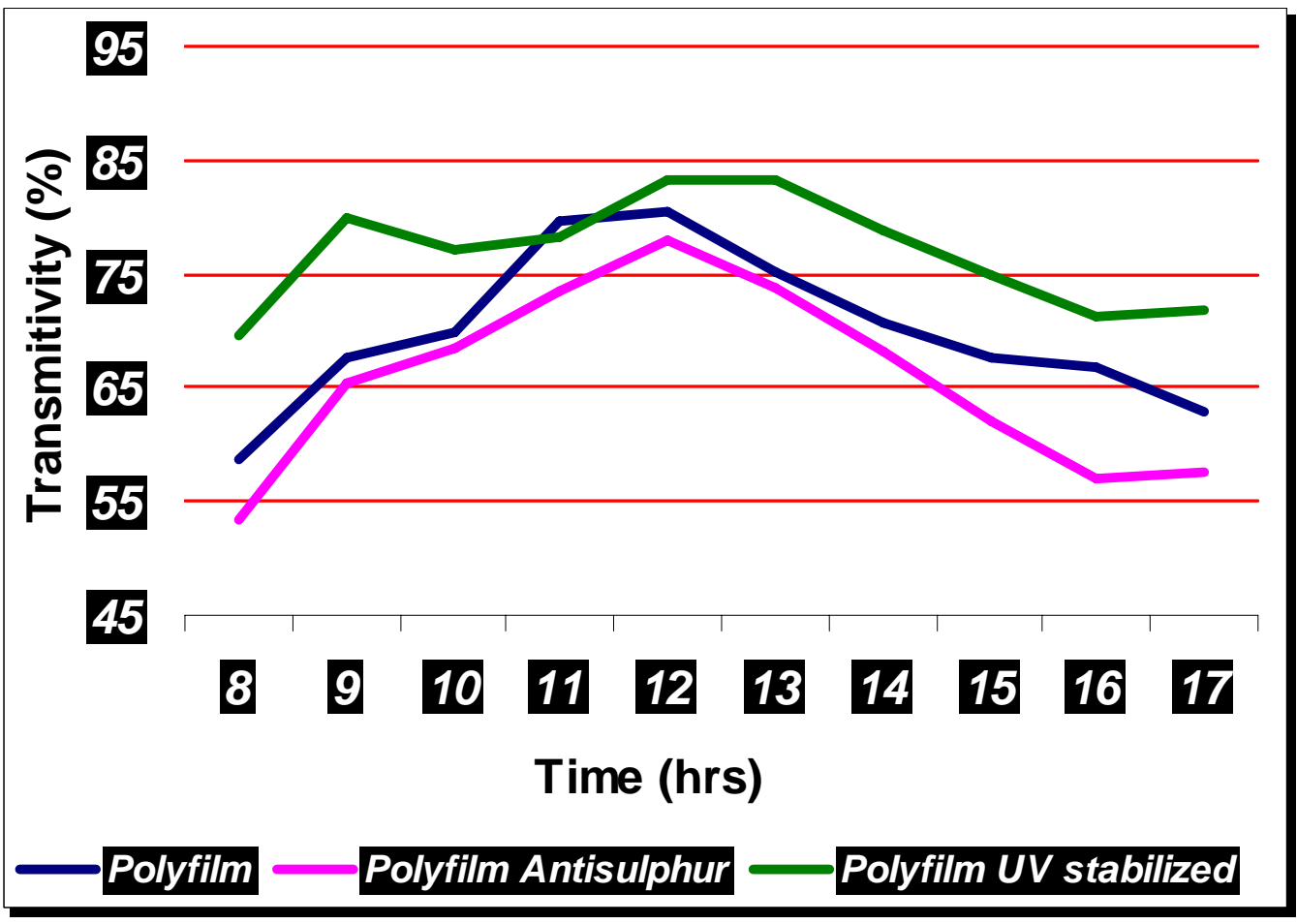

Fig.5. (b) Transmitivity of solar radiation inside poly film. $W$-shape solar still on concrete two channels

No load test

The low cost $W$-shape solar still with area of $2 \mathrm{~m}^{2}(2 \mathrm{~m} \times 1 \mathrm{~m})$ was evaluated for no load test. The maximum average temperature was observed 
during no load test was $49^{\circ} \mathrm{C}$ at 1 p.m. and the same time solar intensity was 553 $\mathrm{W} / \mathrm{m}^{2}$, ambient temperature was $29^{\circ} \mathrm{C}$, and outside relative humidity was 46 per cent. The trend obtained in no load test during performance testing is as shown in Fig 6 and Table 8. It is revealed from Fig 6 that the temperature inside the still increased with solar intensity in morning hours up to 1 P.M., and then started decline as day progressed.

Table: 8. Performance of $W$-shape solar still on concrete two channel with no load test in winter

\begin{tabular}{|c|c|c|c|c|c|}
\hline $\begin{array}{l}\text { Time } \\
\text { (Hrs) }\end{array}$ & $\begin{array}{c}\text { Insolation } \\
\left(\mathrm{W} / \mathrm{m}^{2}\right)\end{array}$ & $\begin{array}{l}\text { Inside } \\
\text { Temp. } \\
\left({ }^{\circ} \mathrm{C}\right)\end{array}$ & $\begin{array}{c}\text { Ambient } \\
\text { Temp. } \\
\left({ }^{\circ} \mathrm{C}\right)\end{array}$ & $\begin{array}{c}\text { Inside } \\
\text { Humidity } \\
(\%)\end{array}$ & $\begin{array}{c}\text { Outside } \\
\text { Humidity } \\
(\%)\end{array}$ \\
\hline 9.00 & 152 & 26 & 21 & 74 & 82 \\
\hline 10.00 & 301 & 34 & 24 & 69 & 71 \\
\hline 11.00 & 454 & 40 & 27 & 59 & 57 \\
\hline 12.00 & 521 & 45 & 28 & 44 & 48 \\
\hline 13.00 & 553 & 49 & 29 & 40 & 46 \\
\hline 14.00 & 502 & 47 & 30 & 36 & 49 \\
\hline 15.00 & 415 & 46 & 29 & 43 & 57 \\
\hline 16.00 & 287 & 44 & 26 & 52 & 61 \\
\hline 17.00 & 163 & 43 & 24 & 55 & 70 \\
\hline 18.00 & 63 & 38 & 23 & 59 & 76 \\
\hline
\end{tabular}

$\longrightarrow$ Inside Temperature (으)

- O utside Rh

$\rightarrow$ Ins olation $(\mathrm{W} / \mathrm{m} 2)$

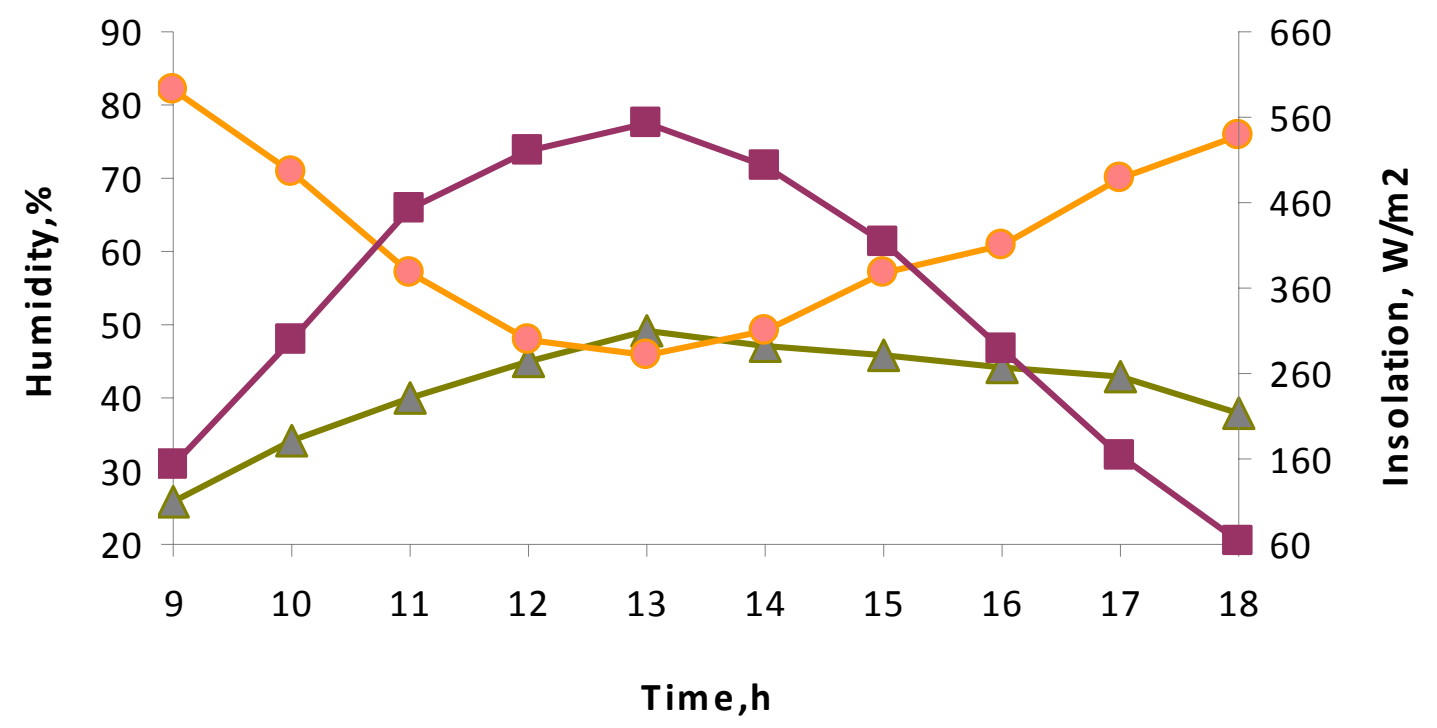

(a) 


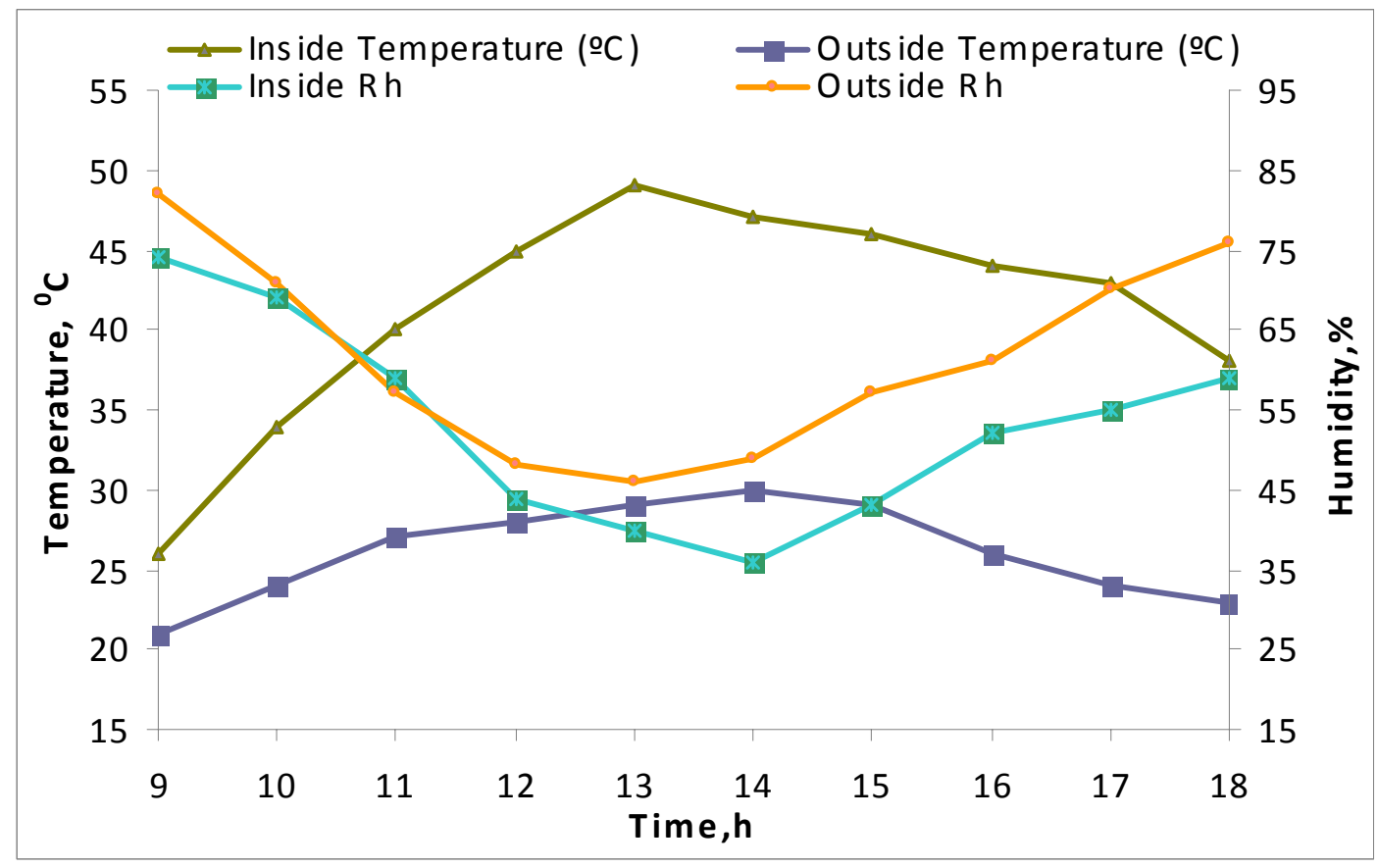

(b)

Fig. 6. Performance of $W$-shape solar still on concrete two channels with no load test in winter

\section{Load test}

$W$-shape solar still with two channels was tested with impounding water depth in cement basin. The hourly cumulative distilled water obtained was observed with solar intensity, ambient temperature, relative humidity and inside temperature. shown in Table 9 and Fig 7.

It was observed from Table 9 that maximum average temperature was observed at 1 p.m., when solar intensity was $523 \mathrm{~W} / \mathrm{m}^{2}$ and ambient temperature was $31.9^{\circ} \mathrm{C}$, ambient R.H. was 48 per cent. Trend observed in load test during performance is shown in Fig 6. It was observed that inside temperature increased as the solar intensity increased and hence rate heat utilization for heating the water was more in noon time and accordingly evaporation was observed more after noon hours and then rate of condensation was increased than noon time as solar intensity decreased. Cumulative distilled water obtained from solar still in summer month was $1274 \mathrm{ml}$ including day and night condensation. It was observed that maximum distillation rate obtained between $3 \mathrm{pm}$ to $4 \mathrm{pm}$ which was highest as $138 \mathrm{ml}$. Average overnight distillation observed in even type solar still unit was $348 \mathrm{ml}$ which was due to higher condensation rate in night. 
Table: 9. Performance of $W$-shape solar still on concrete two channels during load test in summer

\begin{tabular}{|c|c|c|c|c|c|}
\hline $\begin{array}{l}\text { Time } \\
\text { (Hrs) }\end{array}$ & $\begin{array}{l}\text { Insolation } \\
\left(\mathrm{W} / \mathrm{m}^{2}\right)\end{array}$ & $\begin{array}{c}\text { Inside } \\
\text { Temperature } \\
\left({ }^{\circ} \mathrm{C}\right) \\
\end{array}$ & $\begin{array}{c}\text { Outside } \\
\text { Temperature } \\
\left({ }^{\circ} \mathrm{C}\right) \\
\end{array}$ & $\begin{array}{c}\text { Outside } \\
\text { R.H. } \\
\text { (\%) }\end{array}$ & $\begin{array}{c}\text { Cumulative } \\
\text { desalination } \\
(\mathrm{ml})\end{array}$ \\
\hline 8.00 & 103.3 & 23.3 & 25.7 & 75.3 & 0.0 \\
\hline 9.00 & 159.3 & 24.5 & 26.8 & 68.5 & 86.3 \\
\hline 10.00 & 317.5 & 33.8 & 28.2 & 65.6 & 145.0 \\
\hline 11.00 & 376.8 & 43.8 & 28.7 & 61.6 & 230.8 \\
\hline 12.00 & 480.8 & 49.0 & 30.0 & 57.6 & 327.3 \\
\hline 13.00 & 523.1 & 53.8 & 31.4 & 52.1 & 429.5 \\
\hline 14.00 & 499.0 & 55.8 & 31.6 & 50.4 & 537.3 \\
\hline 15.00 & 413.3 & 50.8 & 30.6 & 54.8 & 652.0 \\
\hline 16.00 & 336.3 & 45.3 & 29.5 & 59.9 & 790.8 \\
\hline 17.00 & 228.3 & 41.1 & 28.8 & 63.8 & 862.0 \\
\hline 18.00 & 150.5 & 36.3 & 28.0 & 67.4 & 925.0 \\
\hline \multicolumn{5}{|c|}{$\begin{array}{l}\text { Overnight distillation up to } 8.00 \text { a.m. } \\
\text { Total }\end{array}$} & $\begin{array}{c}348.8 \\
1273.8\end{array}$ \\
\hline
\end{tabular}

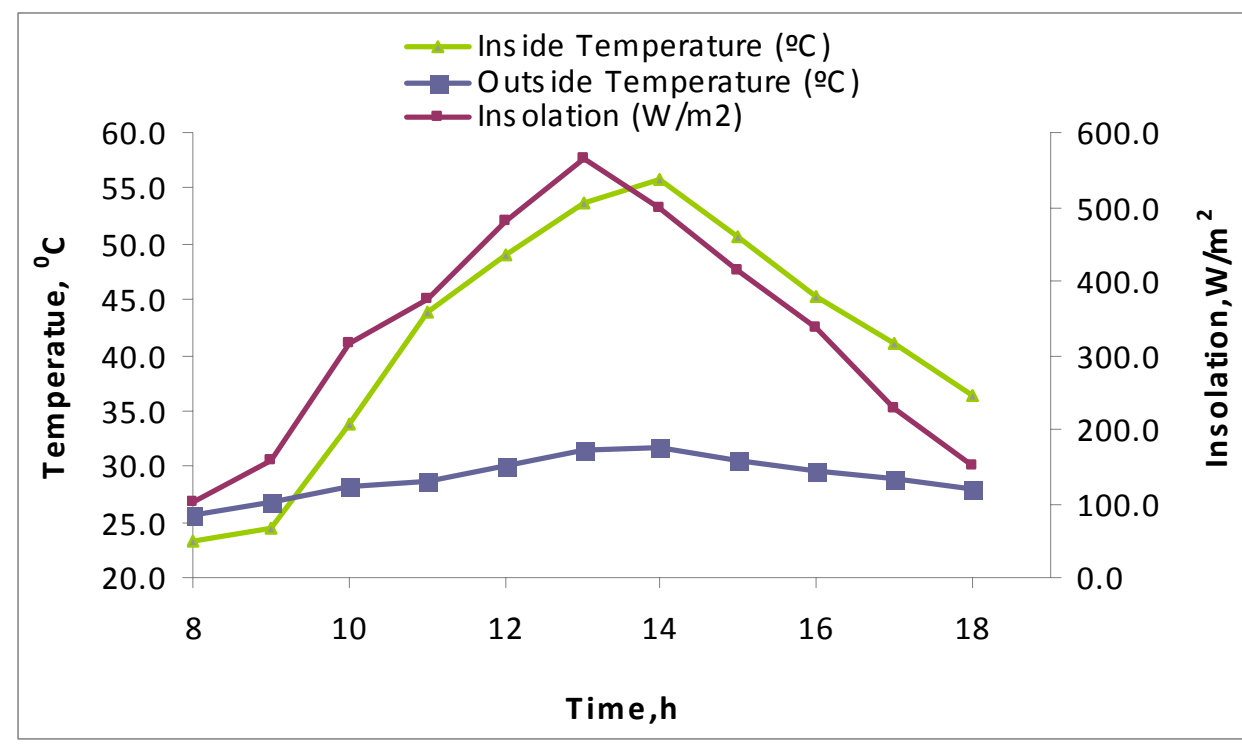

(a) 


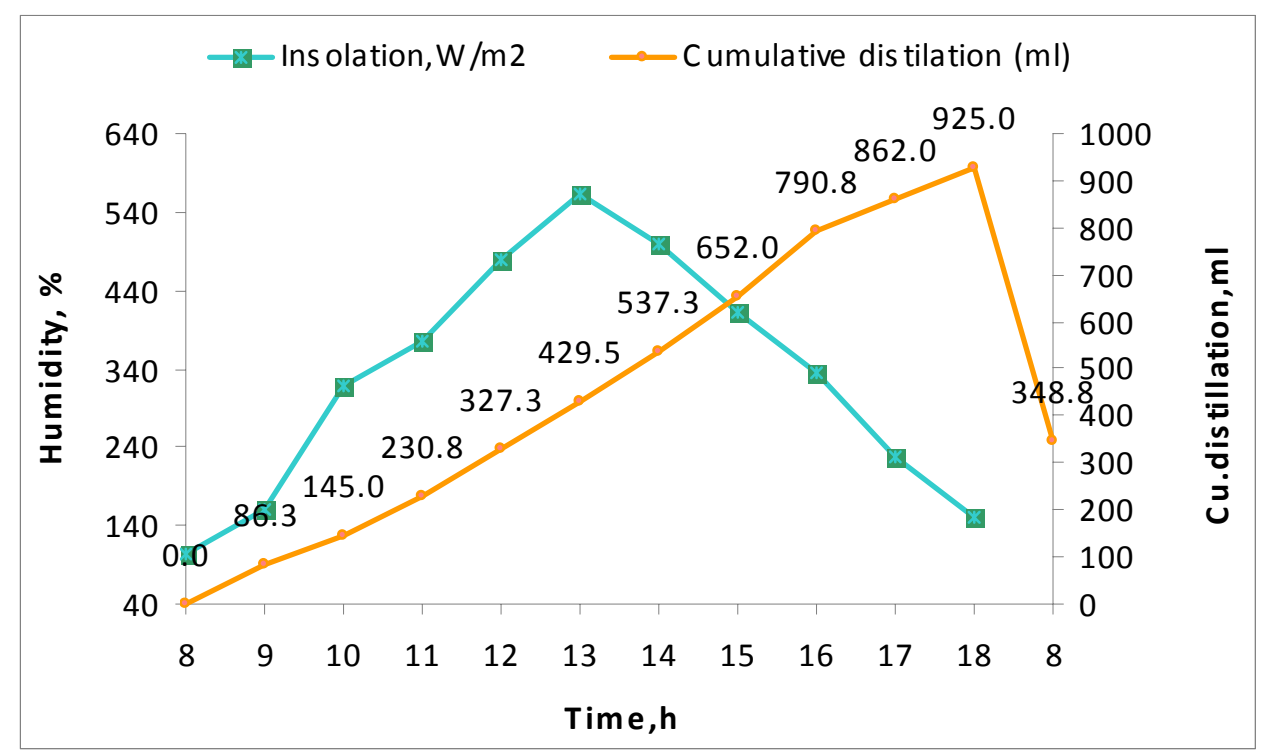

(b)

Fig. 7. Performance of $W$-shape solar still on concrete two channels during load test in summer

The maximum cumulative distillation obtained in summer season was $1273 \mathrm{ml}$, solar intensity played vital role in rate and quantity of distillation through the plant.

\section{W- shape solar still on soil two channels} No load

$W$-shape solar still with two channels was erected on ground and the collected distilled water with two channels was periodically observed along with solar energy, ambient temperature. Inside and outside humidity. The still was evaluated in summer for no load. In summer, maximum inside temperature reached in solar still was $45.1{ }^{\circ} \mathrm{C}$ where ambient temperature, solar radiation and relative humidity were found as 35.2,612 and35.2 respectively shown in Table 10 and Fig.8. 
Table: 10. Performance of $W$-shape solar still on ground two channels during no load test in summer

\begin{tabular}{cccccc}
\hline $\begin{array}{c}\text { Time } \\
(\mathbf{H r s})\end{array}$ & $\begin{array}{c}\text { Insolation } \\
\left(\mathbf{W} / \mathbf{m}^{2}\right)\end{array}$ & $\begin{array}{c}\text { Inside } \\
\mathbf{T e m p e r a t u r e} \\
\left({ }^{\circ} \mathbf{C}\right)\end{array}$ & $\begin{array}{c}\text { Outside } \\
\mathbf{T e m p e r a t u r e} \\
\left({ }^{\circ} \mathbf{C}\right)\end{array}$ & $\begin{array}{c}\text { Inside } \\
\mathbf{R h}\end{array}$ & $\begin{array}{c}\text { Outside } \\
\mathbf{R h}\end{array}$ \\
\hline 8.00 & 208 & 28.2 & 26.6 & 70.5 & 72.1 \\
9.00 & 268 & 33.1 & 29.9 & 59.2 & 65.4 \\
10.00 & 355 & 35.8 & 31.5 & 52.4 & 61.5 \\
11.00 & 487 & 45.4 & 33.6 & 43.8 & 58.7 \\
12.00 & 549 & 47.2 & 34.5 & 37.2 & 55.2 \\
13.00 & 612 & 45.1 & 35.2 & 35.2 & 52.9 \\
14.00 & 502 & 46.8 & 33 & 45.4 & 50.4 \\
15.00 & 355 & 44.4 & 32.2 & 51.7 & 54.6 \\
16.00 & 306 & 44.2 & 30.2 & 52.6 & 57.8 \\
17.00 & 177 & 43.4 & 31 & 51.4 & 61.4 \\
18.00 & 102 & 42.1 & 28.5 & 55 & 62.5 \\
\hline
\end{tabular}

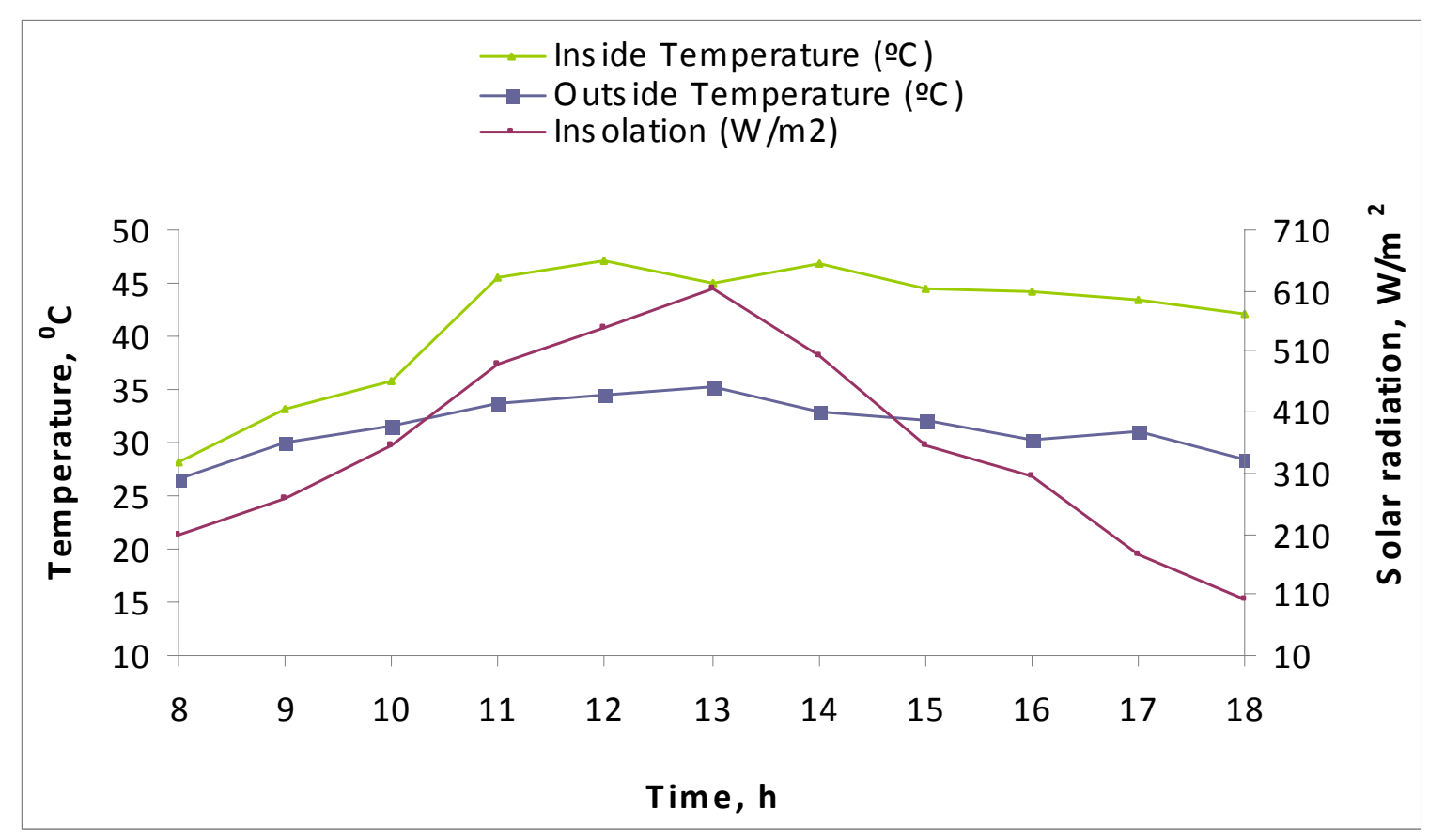

(a) 


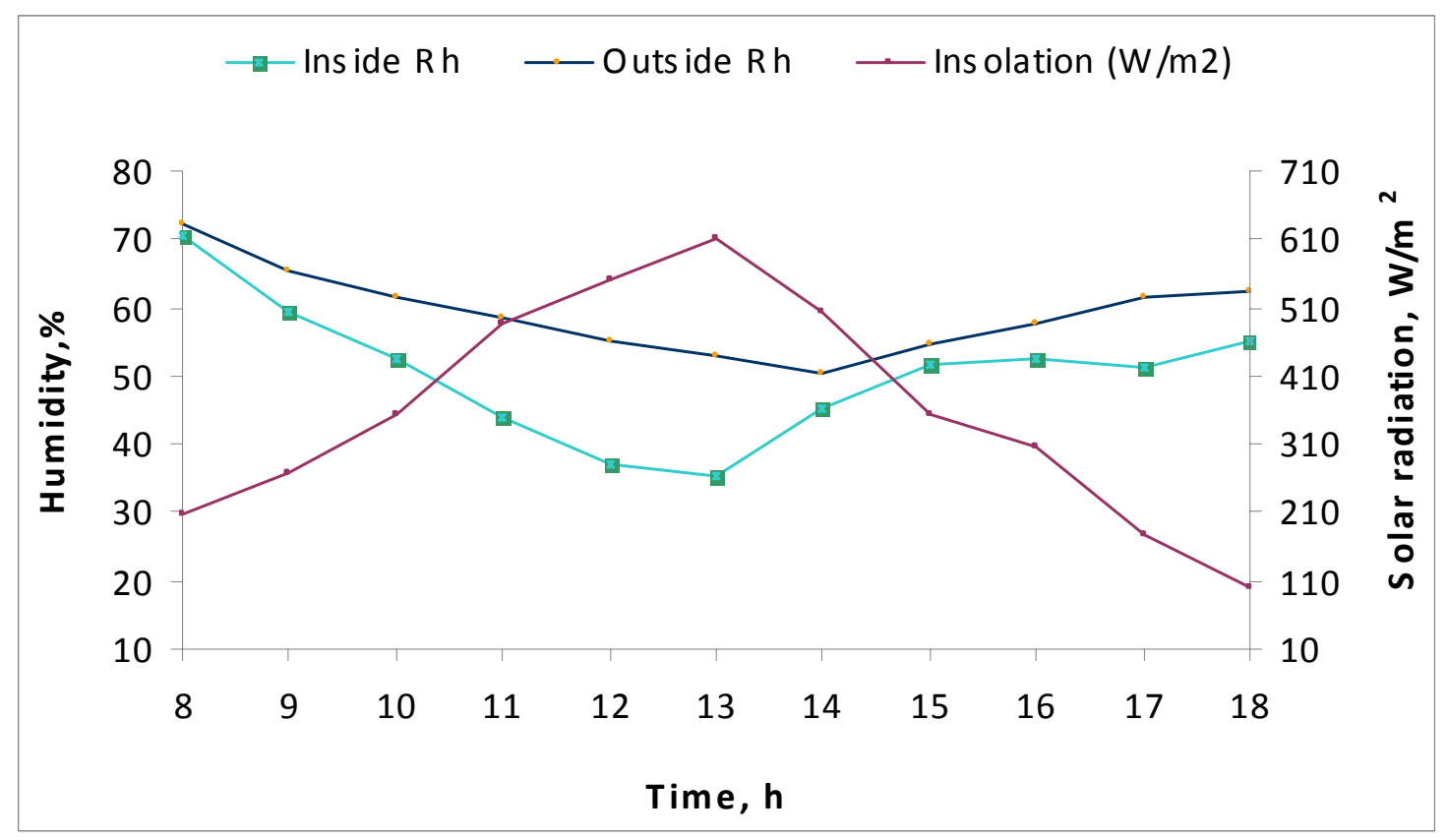

(b)

Fig. 8. Performance of $W$-shape solar still on ground two channels during

\section{With Load}

\section{no load test in summer}

$W$ shape solar still erected on ground with a provision of two channels for collections was evaluated for winter and summer depicted in Table 11 and Fig.9 and Table 12 and Fig.10 respectively. In winter maximum distilled water collected from 24 hour was only $950 \mathrm{ml}$ which was very less comparing to distilled water obtained in summer as $1936.5 \mathrm{ml}$ from same unit. 
Table: 11. Performance of $W$-shape solar still on ground two channels during

load test in winter

\begin{tabular}{|c|c|c|c|c|c|c|c|c|}
\hline $\begin{array}{c}\text { Time } \\
\mathrm{h} \\
\end{array}$ & $\begin{array}{c}\text { Insolation } \\
(\mathrm{W} / \mathrm{m} 2)\end{array}$ & $\begin{array}{c}\text { Inside Temp. } \\
\left({ }^{\circ} \mathrm{C}\right)\end{array}$ & $\begin{array}{c}\text { Outside } \\
\text { Temp. } \\
\left.{ }^{\circ} \mathrm{C}\right)\end{array}$ & $\begin{array}{c}\text { Inside } \\
\text { Rh } \\
(\%) \\
\end{array}$ & $\begin{array}{c}\text { Outside } \\
\text { Rh } \\
(\%) \\
\end{array}$ & $\begin{array}{c}\text { Cu.dist } \\
\text {.-pot-1 } \\
\text { (ml) }\end{array}$ & $\begin{array}{l}\text { Cu.dist.- } \\
\text { Pot-2(ml) }\end{array}$ & $\begin{array}{c}\text { Total } \\
\text { Cu.dist. } \\
\text { (ml) }\end{array}$ \\
\hline 8.0 & 114.3 & 22.4 & 19.8 & 76.6 & 75.6 & 0.0 & 0.0 & 0.0 \\
\hline 9.0 & 232.0 & 29.5 & 21.3 & 73.8 & 69.8 & & & \\
\hline 10.0 & 362.5 & 34.6 & 23.1 & 58.1 & 62.3 & 58.8 & 64.4 & 123.1 \\
\hline 11.0 & 474.4 & 38.2 & 28.6 & 53.7 & 52.3 & & & \\
\hline 12.0 & 522.1 & 44.7 & 30.5 & 48.8 & 49.5 & 82.5 & 95.6 & 178.1 \\
\hline 13.0 & 538.8 & 44.2 & 29.7 & 50.1 & 48.6 & & & \\
\hline 14.0 & 445.8 & 45.9 & 29.2 & 47.6 & 49.5 & 130.6 & 143.8 & 274.4 \\
\hline 15.0 & 362.9 & 40.8 & 28.4 & 47.0 & 51.5 & & & \\
\hline 16.0 & 284.1 & 40.1 & 28.0 & 50.1 & 54.4 & 183.8 & 205.6 & 389.4 \\
\hline 17.0 & 132.1 & 35.4 & 25.9 & 58.0 & 56.3 & & & \\
\hline 18.0 & 40.0 & 30.1 & 24.7 & 67.5 & 65.9 & 219.4 & 238.8 & 458.1 \\
\hline \multicolumn{6}{|c|}{ Overnight distillation up to 8.00 a.m. } & 239.2 & 252.8 & $\begin{array}{c}491.9 \\
950\end{array}$ \\
\hline
\end{tabular}

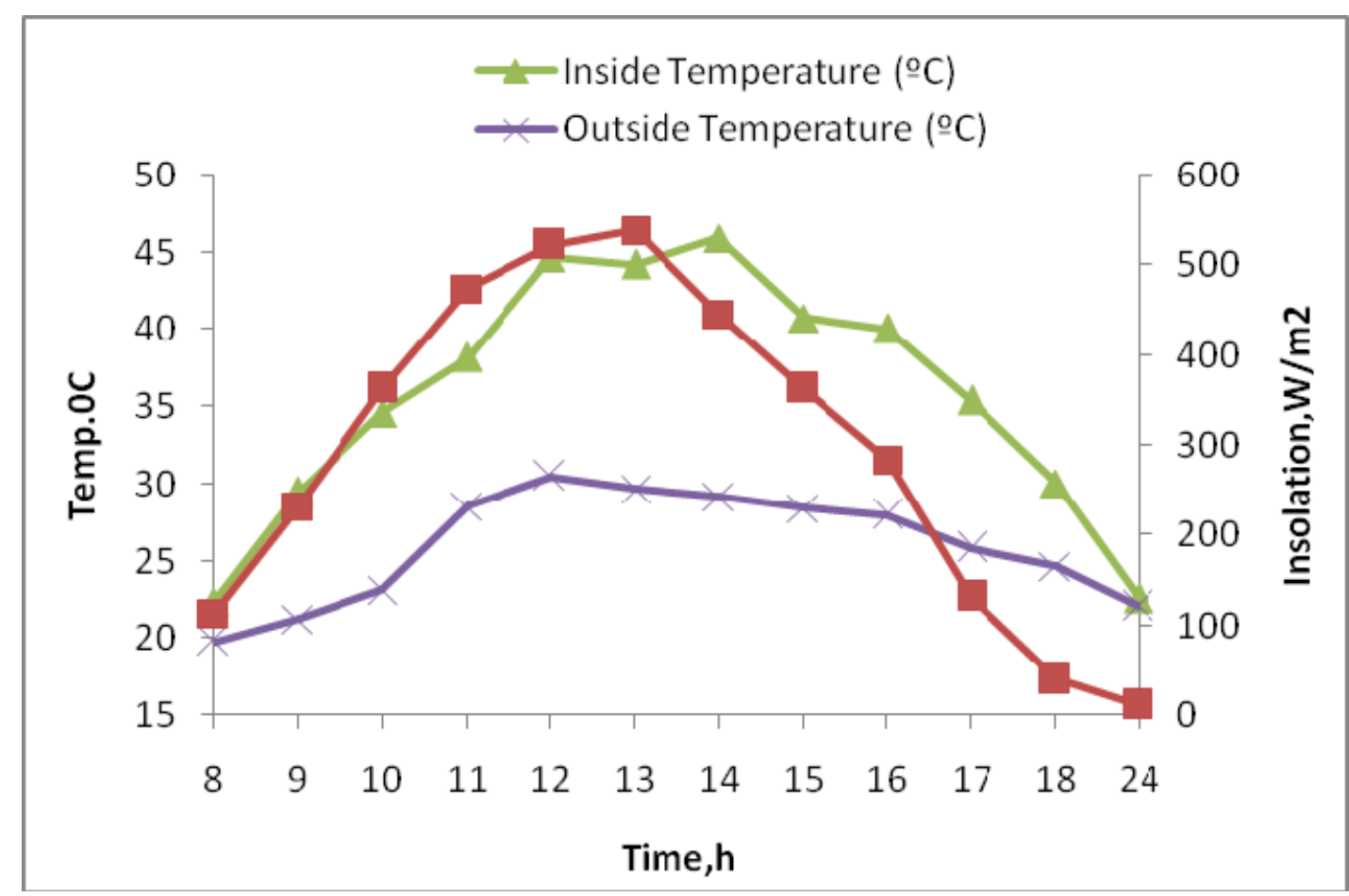

(a) 


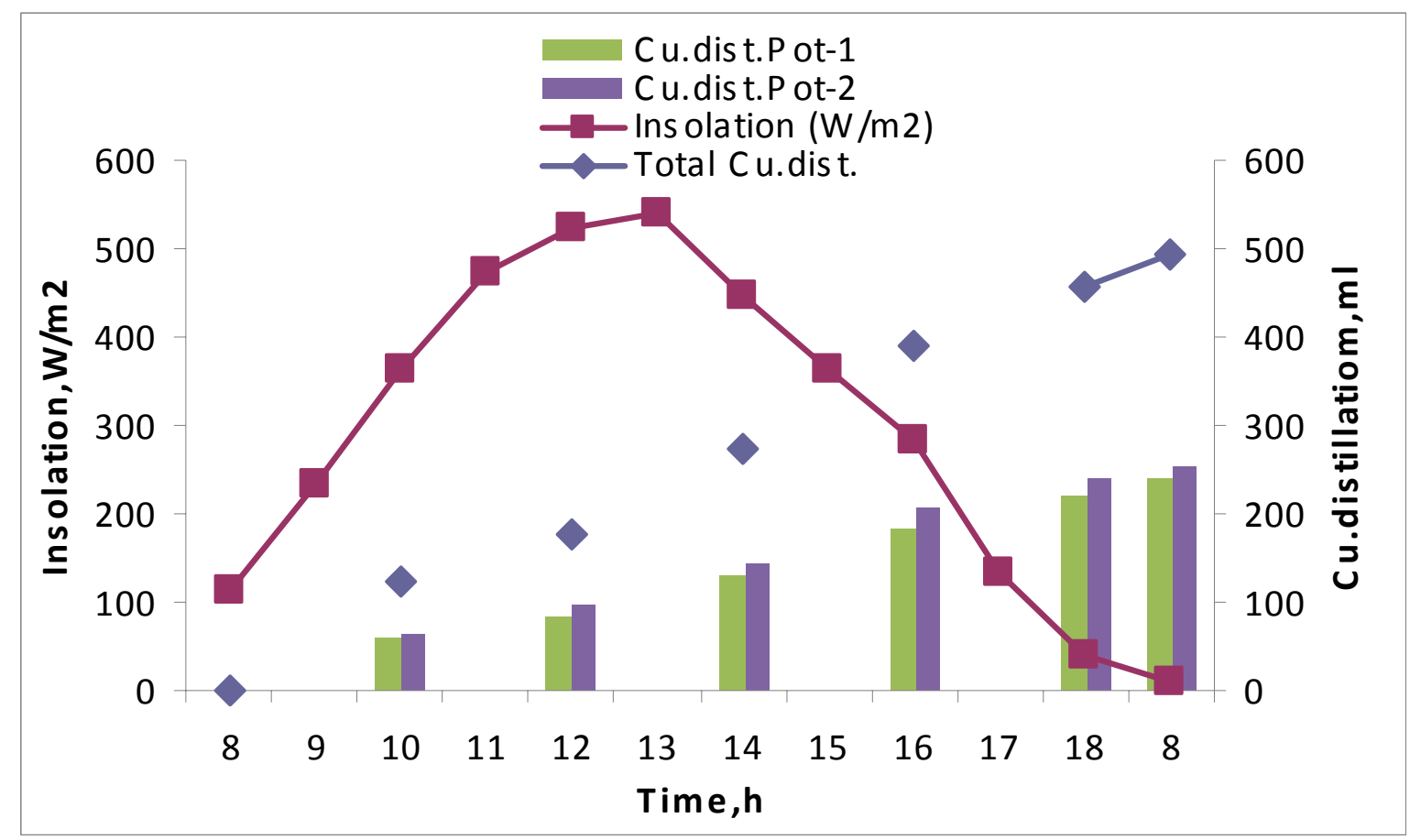

(b)

Fig. 9. Performance of $W$-shape solar still on ground two channels during load test in winter

Table: 12. Performance of $W$-shape solar still on ground two channels during load test in summer

\begin{tabular}{cccccc}
\hline Time & $\begin{array}{c}\text { Insolation } \\
\left(\mathbf{W} / \mathbf{m}^{\mathbf{2}} \mathbf{)}\right.\end{array}$ & $\begin{array}{c}\text { Inside } \\
\text { Temperature } \\
\left({ }^{\circ} \mathbf{C}\right)\end{array}$ & $\begin{array}{c}\text { Outside } \\
\text { Temperature } \\
\left.\mathbf{(} \mathbf{}^{\circ} \mathbf{C}\right)\end{array}$ & $\begin{array}{c}\text { Inside } \\
\mathbf{R h} \\
\mathbf{( \% )}\end{array}$ & $\begin{array}{c}\text { Cumulative } \\
\text { distilation } \\
(\mathbf{m l})\end{array}$ \\
\hline 8 & 195.0 & 22.2 & 27.3 & 75.1 & 0.0 \\
9 & 371.0 & 26.2 & 28.4 & 72.6 & 122.5 \\
10 & 438.0 & 34.7 & 30.7 & 64.9 & 237.8 \\
11 & 516.0 & 47.4 & 32.3 & 59.4 & 358.3 \\
12 & 568.0 & 58.2 & 34.0 & 50.8 & 522.8 \\
13 & 600.0 & 60.7 & 34.6 & 47.0 & 696.5 \\
14 & 555.0 & 52.4 & 33.8 & 50.0 & 847.8 \\
15 & 420.3 & 43.8 & 32.3 & 52.9 & 987.0 \\
16 & 364.0 & 40.0 & 31.1 & 54.0 & 1136.8 \\
17 & 291.0 & 36.6 & 29.7 & 57.6 & 1229.8 \\
18 & 140.8 & 33.9 & 28.6 & 59.7 & 1299.0 \\
& Overnight distillation up to 8.00 a.m. & & 637.5 \\
& \multicolumn{5}{c}{ Total } \\
\hline
\end{tabular}




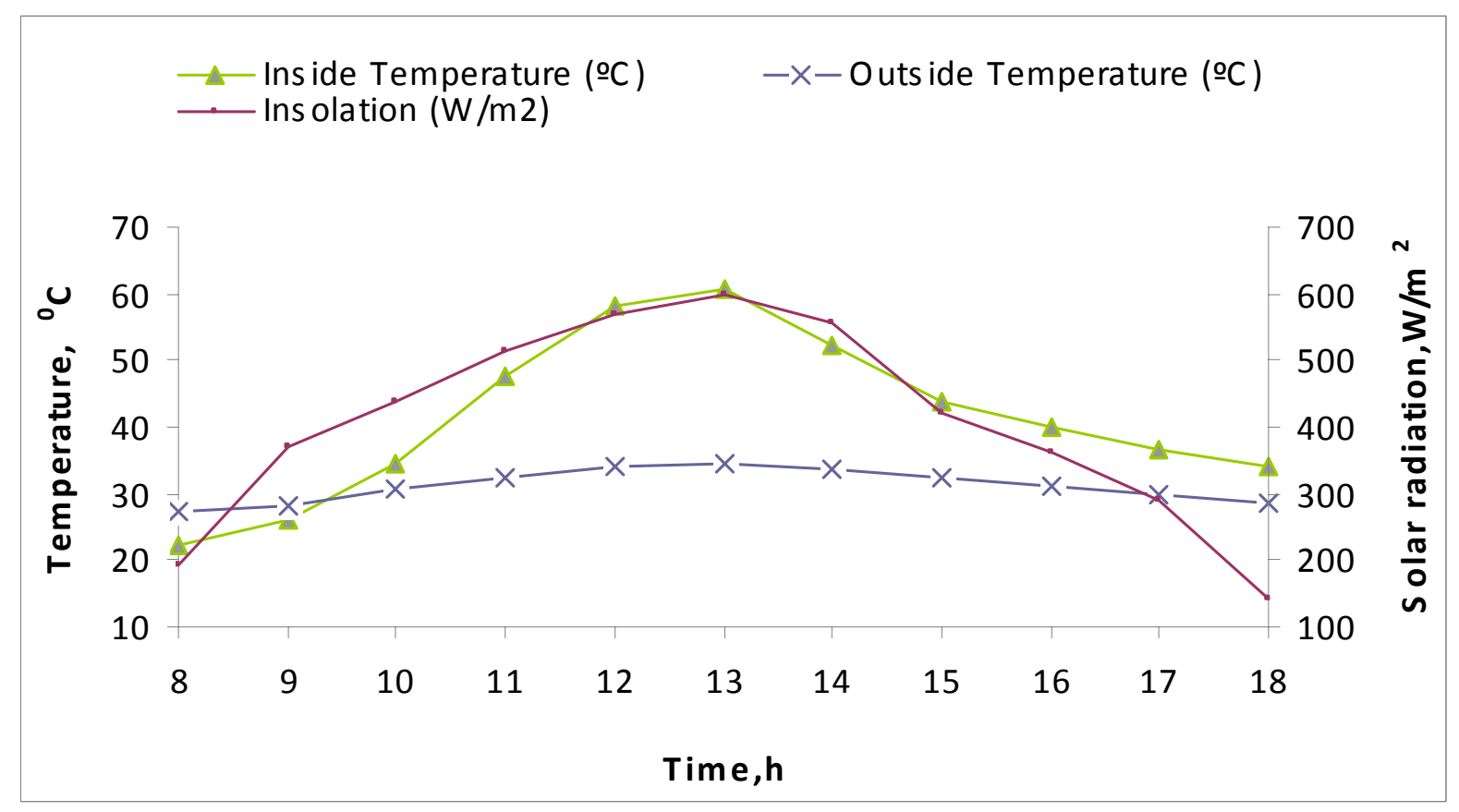

(a)

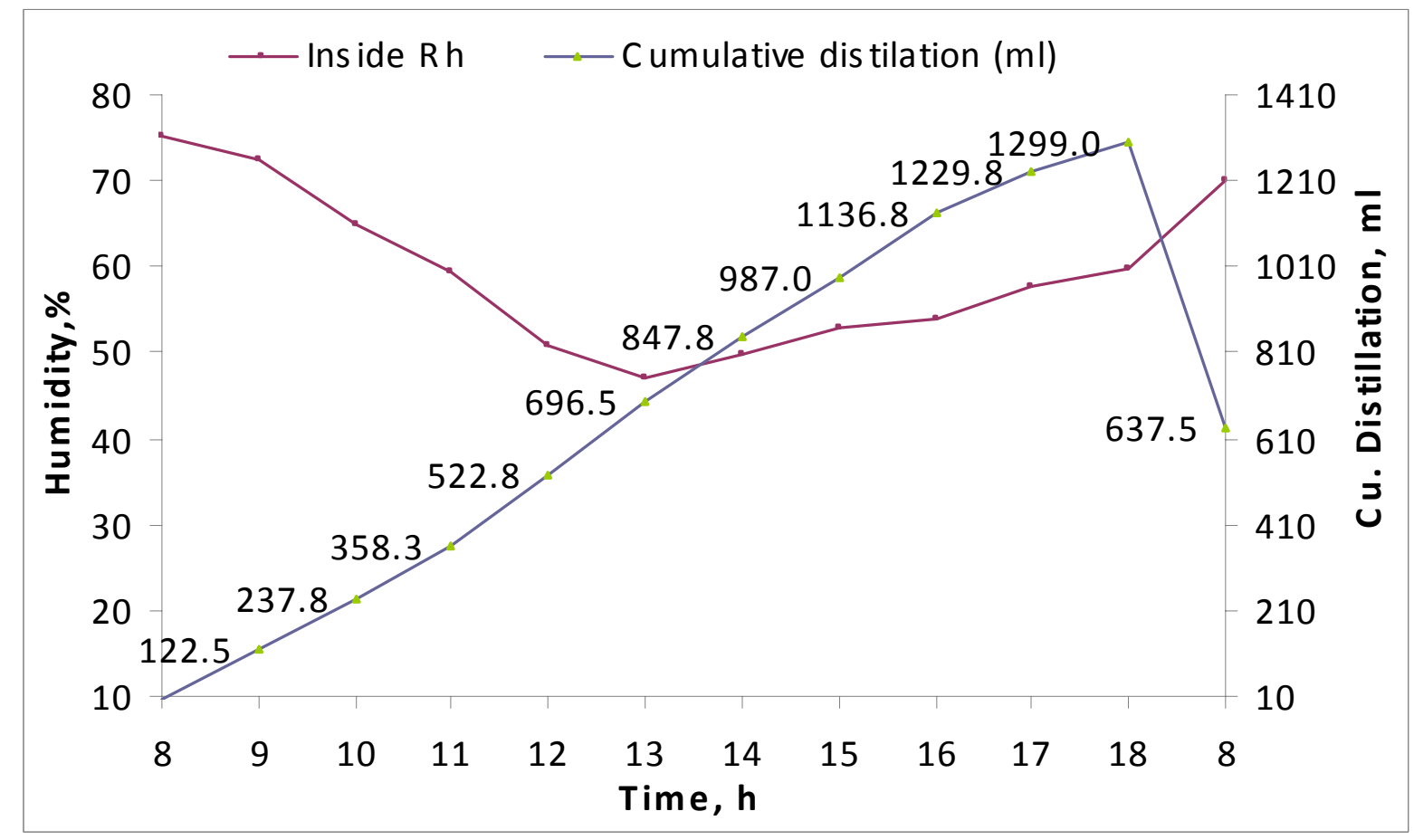

(b)

Fig. 10. Performance of $W$-shape solar still on ground two channels during load test in summer 
Single side ( $L$ - shape one channel) solar still on soilWith Load

$L$ shape solar still was erected on ground with single channel for collection provision. The still was evaluated in winter for load condition. In winter, maximum inside temperature reached in solar still was $63.8{ }^{\circ} \mathrm{C}$ where ambient temperature, solar radiation and relative humidity were found as 30.3, $540 \mathrm{~W} / \mathrm{m}^{2}$ and $51.8 \%$ respectively shown in Table 13 and Fig. 11 . The Maximum distilled water collected from this unit in winter was only $850 \mathrm{ml}$ in 24 hours.

Table: 13. Performance of $L$ type still on ground during load test in winter

\begin{tabular}{cccccc}
\hline Time & $\begin{array}{c}\text { Insolation } \\
\left(\mathbf{W} / \mathbf{m}^{\mathbf{2}}\right)\end{array}$ & $\begin{array}{c}\text { Inside } \\
\text { Temperature } \\
\left({ }^{\circ} \mathbf{C}\right)\end{array}$ & $\begin{array}{c}\text { Outside } \\
\text { Temperature } \\
\left({ }^{\circ} \mathbf{C}\right)\end{array}$ & $\begin{array}{c}\text { Inside } \\
\mathbf{R h}\end{array}$ & $\begin{array}{c}\text { Cumulative } \\
\text { distilation } \\
(\mathbf{m l})\end{array}$ \\
\hline 8 & 70.0 & 21.5 & 23.4 & 72.4 & 0.0 \\
9 & 229.0 & 28.9 & 25.2 & 65.2 & 12.5 \\
10 & 324.0 & 33.0 & 27.5 & 60.3 & 32.5 \\
11 & 453.5 & 42.4 & 28.6 & 46.4 & 72.5 \\
12 & 493.0 & 54.4 & 29.5 & 44.0 & 130.0 \\
13 & 540.0 & 60.5 & 30.3 & 51.8 & 197.5 \\
14 & 495.0 & 63.8 & 29.0 & 51.0 & 230.0 \\
15 & 416.5 & 50.1 & 28.7 & 39.8 & 282.5 \\
16 & 317.5 & 49.7 & 27.7 & 48.3 & 370.0 \\
17 & 218.5 & 46.0 & 26.5 & 49.1 & 430.0 \\
18 & 88.5 & 40.2 & 23.4 & 51.7 & 475.0 \\
\multicolumn{5}{c}{ Overnight distillation up to 8.00 a.m. } \\
\hline \multicolumn{5}{c}{ Total } \\
\hline \multicolumn{5}{c}{}
\end{tabular}




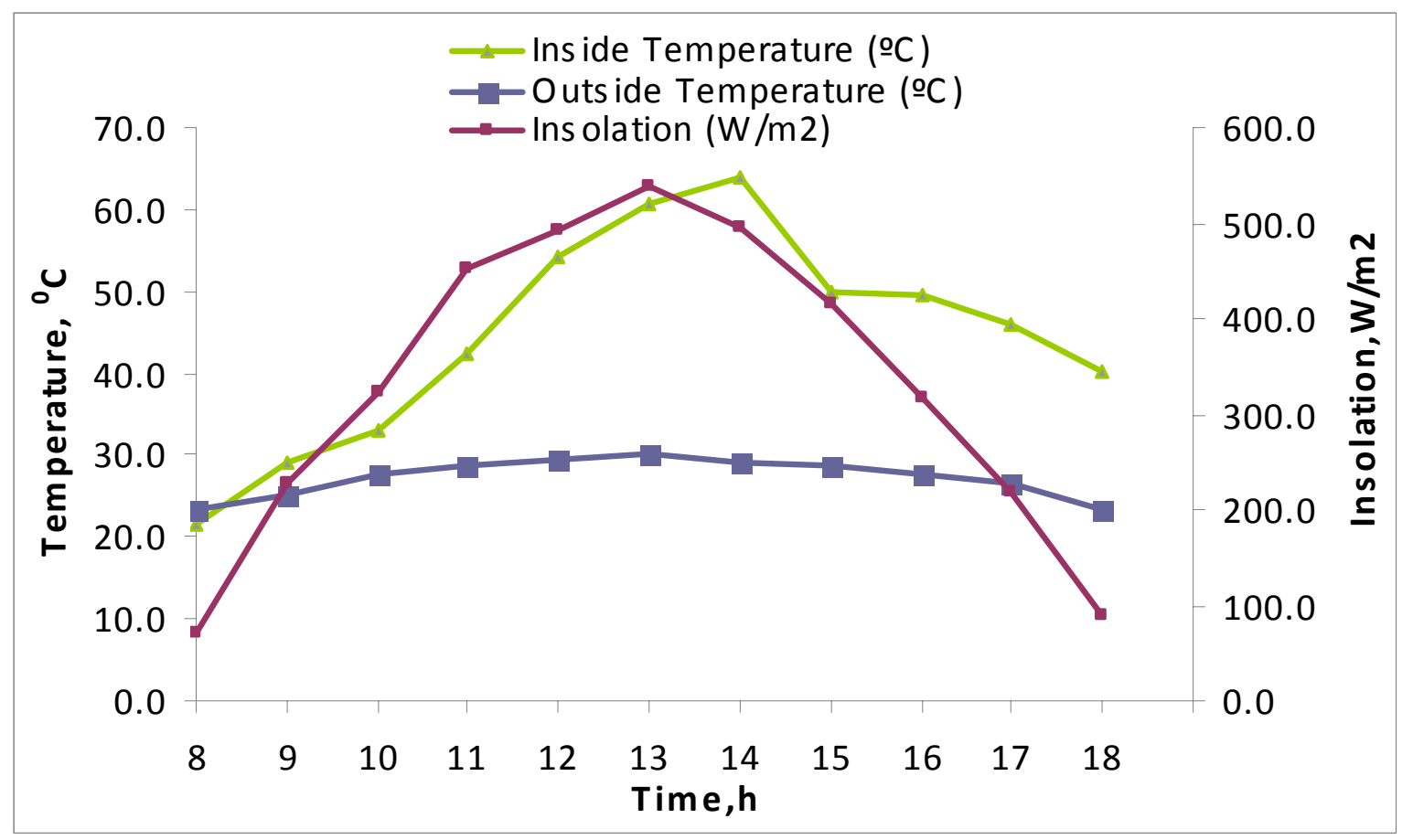

(a)

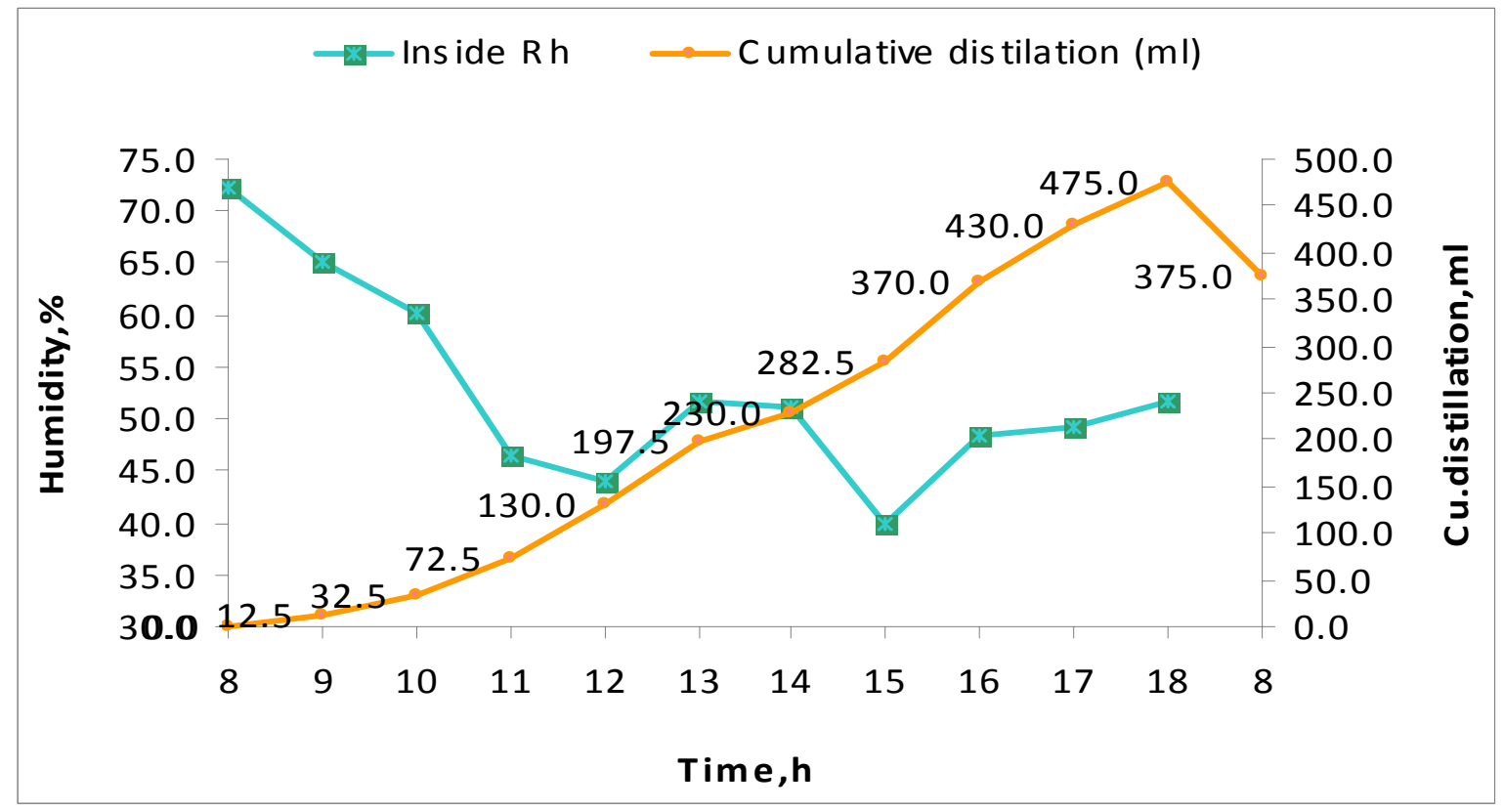

(b)

Fig. 11. Performance of $L$ type solar still on ground during load test in winter

\section{W- shape solar still on ground three channel}

\section{Load in winter}

W-shape solar still erected on ground with three channels for collection was evaluated in winter and summer for load test and respective data is depicted in Table 14 and Fig 12. Maximum distilled water collected from solar still erected 
on soil with three channels was $1633 \mathrm{ml} /$ day. The maximum average solar radiation available in winter during study was $556 \mathrm{~W} / \mathrm{m}^{2}$ and maximum average inside temperature, inside relative humidity were found as $46{ }^{\circ} \mathrm{C}, 73.8 \%$ respectively. By providing one extra collection channel surrounding the bottom sides of solar still, $65 \%$ increased was observed in total cumulative distillation.

Table: 14. Performance of $W$-shape solar still on ground three channels during load test in winter

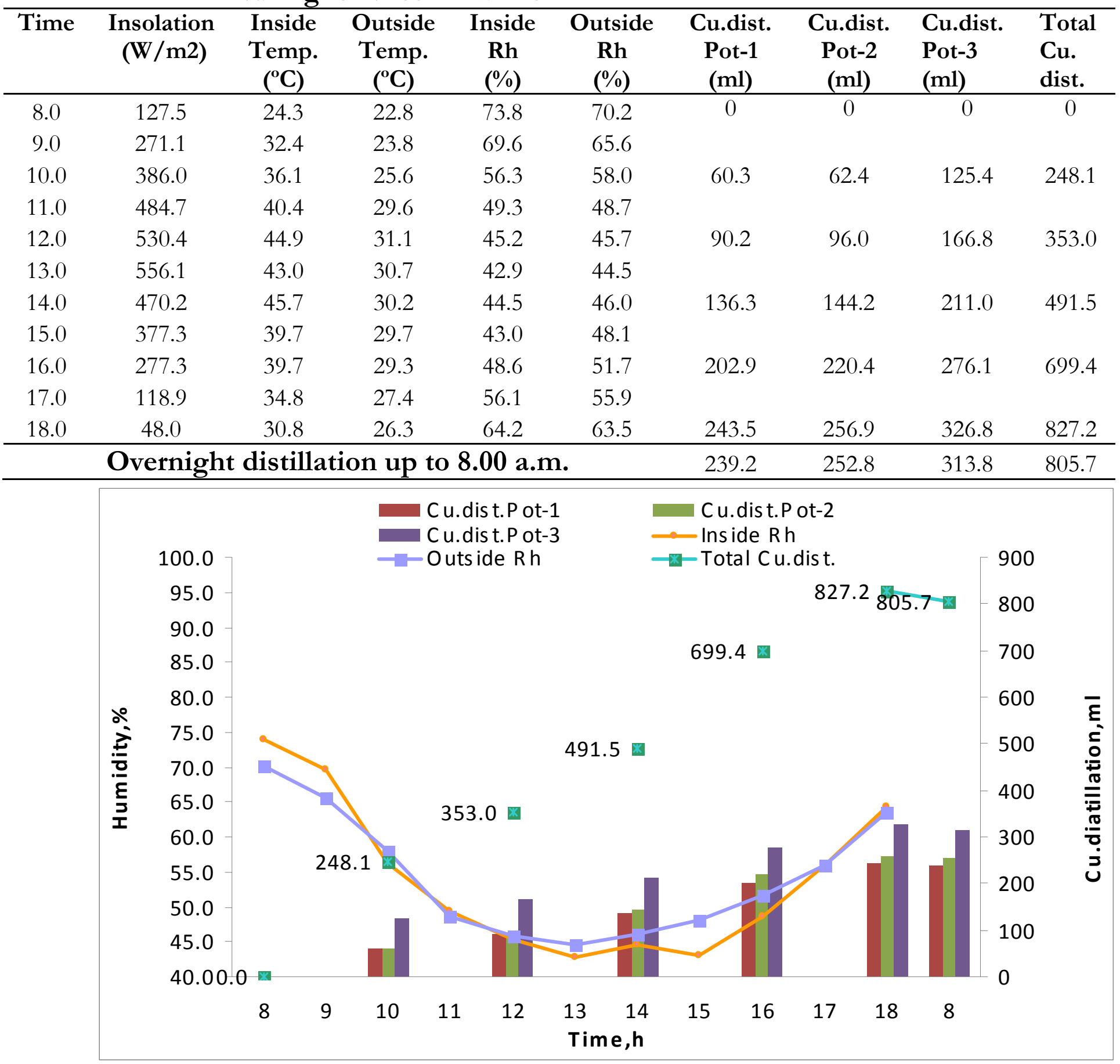




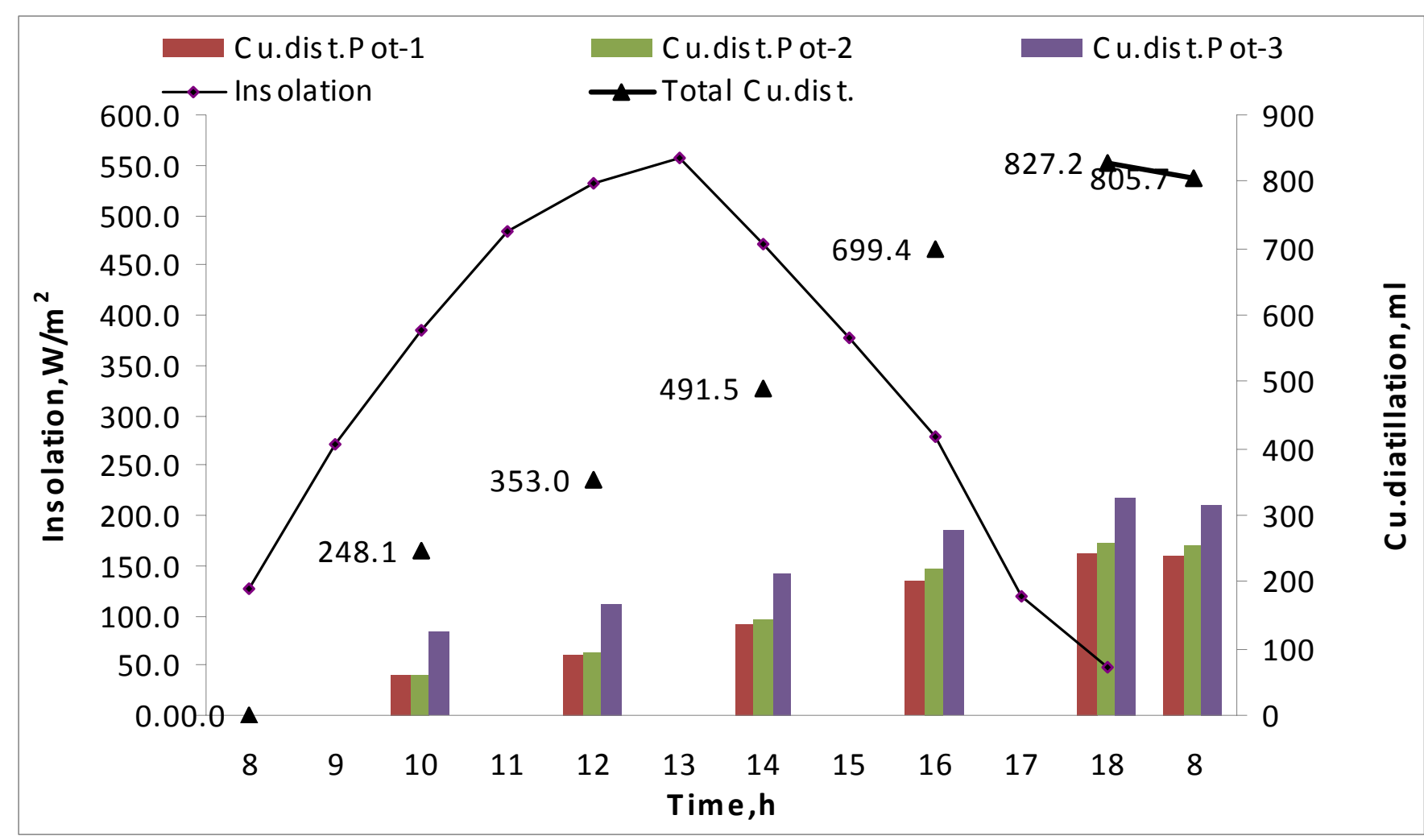

(b)

Fig. 12. Performance evaluation of $W$-shape solar still on ground with three channels in winter

\section{Load test in summer}

$W$-shape solar still erected on ground with three channel provision was evaluated in summer for load test and respective data are depicted in Table 15 and Fig 13. Maximum distilled water collected from solar still erected on ground with three channels was $2575 \mathrm{ml} /$ day. By providing one extra collection channel surrounding the bottom side of solar still, $55 \%$ rise was observed in total cumulative distillation in summer.

Table: 15. Performance of $W$-shape solar still on ground with three channels during load test in summer

\begin{tabular}{ccccccc}
\hline Time & $\begin{array}{c}\text { Insolation } \\
(\mathbf{W} / \mathbf{m} 2)\end{array}$ & $\begin{array}{c}\text { Outside } \\
\text { Temp. } \\
\left({ }^{\circ} \mathbf{C}\right)\end{array}$ & $\begin{array}{c}\text { Cu.dist. } \\
\text { Pot-1 } \\
(\mathbf{m l})\end{array}$ & $\begin{array}{c}\text { Cu.dist. } \\
\text { Pot-2 } \\
(\mathbf{m l})\end{array}$ & $\begin{array}{c}\text { Cu.dist. } \\
\text { Pot-3 } \\
(\mathbf{m l})\end{array}$ & $\begin{array}{c}\text { Total } \\
\text { Cu. } \\
\text { dist. }\end{array}$ \\
\hline 8.00 & 193.2 & 28.7 & 0.0 & 0.0 & 0.0 & 0.0 \\
10.00 & 490.8 & 31.8 & 73.0 & 53.0 & 122.0 & 248.0 \\
12.00 & 561.6 & 32.2 & 164.0 & 120.0 & 243.0 & 527.0 \\
14.00 & 510.6 & 32.5 & 349.0 & 292.0 & 458.0 & 1099.0 \\
\hline
\end{tabular}




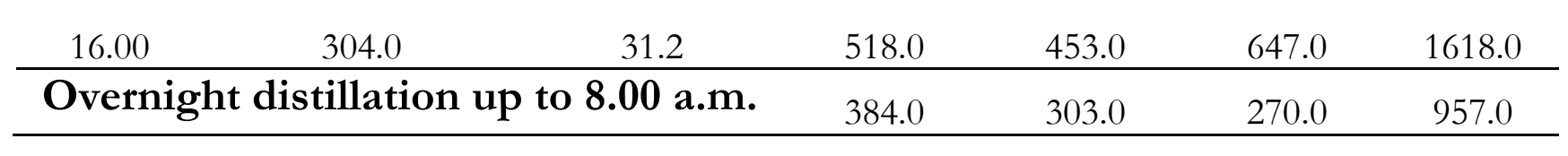

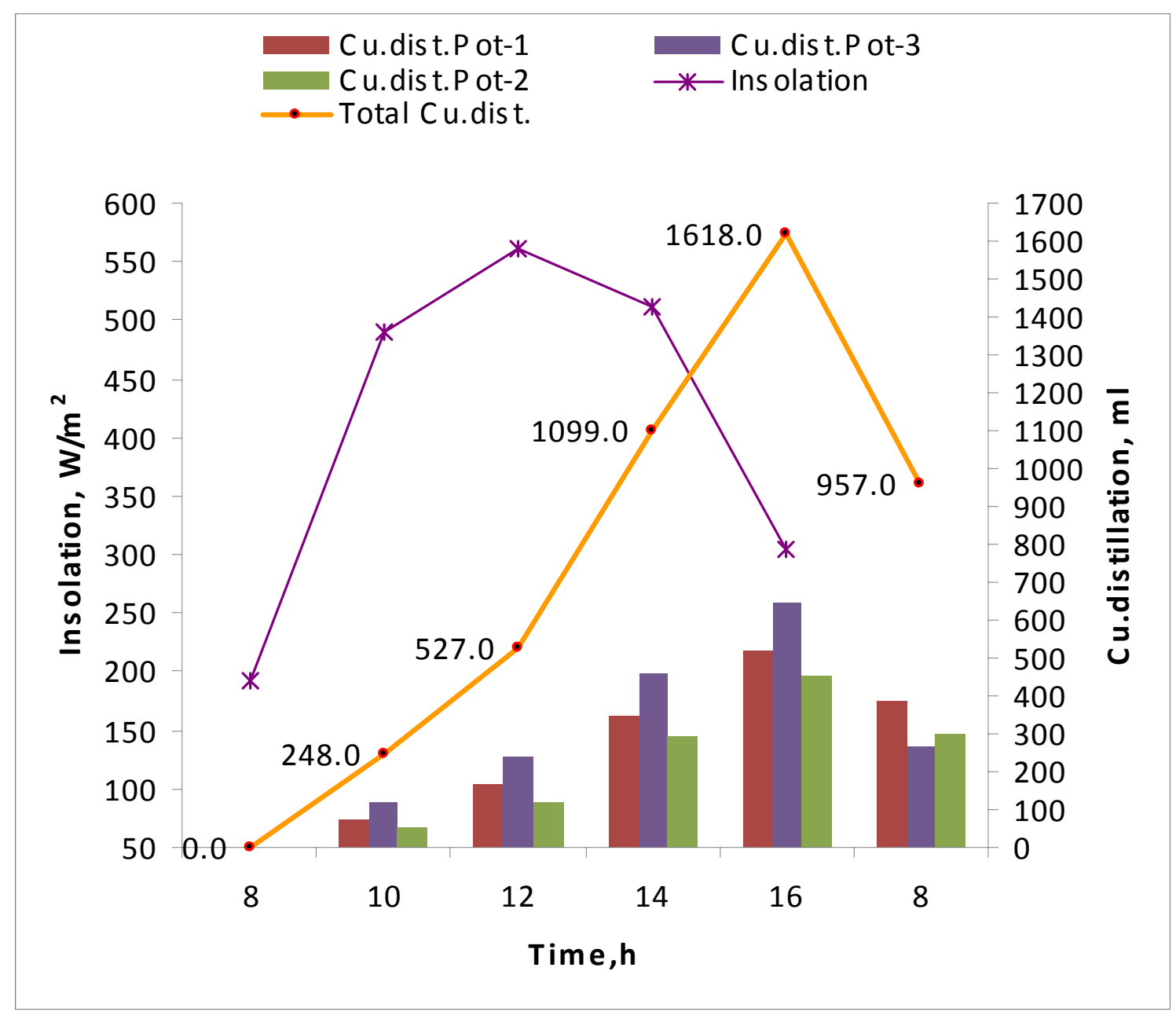

(a) 


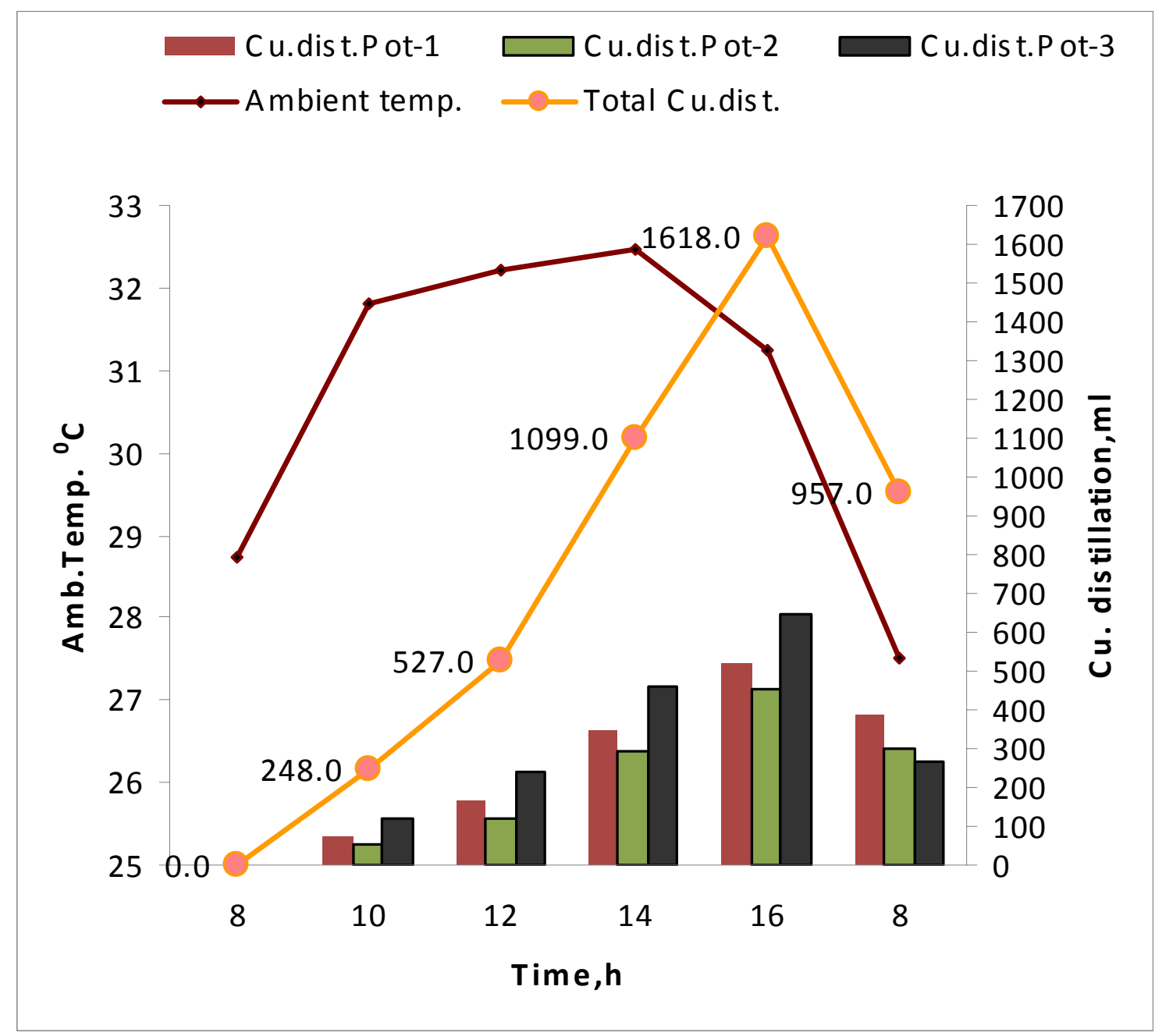

Fig. 13. Performance evaluation of $W$-shape solar still on ground with three channel in summer

\section{Comparison of different solar distillation units}

After evaluating the different solar stills, their results of cumulative distillation are compared and depicted in Table16. Comparatively more distilled water was obtained from the solar still having an area of $1 \mathrm{~m}^{2}$ fabricated in fiber or metal body with glass glazing like single slope ,double slope and wick type solar still. From Table 16 shows that average maximum temperature and humidity was more in double slope, single slope and wick type solar still and hence the average quantity of distilled water obtained as $1350 \mathrm{ml} /$ day, 1550 $\mathrm{ml} /$ day and $2450 \mathrm{ml} /$ day respectively. Reason behind the maximum output from single slope ,double slope and wick type solar still was proper insulation and glass glazing where as another solar stills made with plastic wrapping over the frame which itself acted as body of solar still and glazing for maximum energy collection. 
When jute cloth was spread above the absorber in wick type solar still, it increased the evaporation rate than single and double slope still and hence comparatively more distilled water was obtained in wick type solar still.

This plastic made up of $W$-shape solar still provided with 3 channels produce maximum distilled water as $2104 \mathrm{ml} /$ day where as $W$-shape solar still with two channels erected on concrete and ground produced only $1012 \mathrm{ml} /$ day and $1443 \mathrm{ml} /$ day respectively. $\mathrm{L}$ shape solar still produce average distilled water was $925 \mathrm{ml} /$ day which was very low among the all type of solar still.

Comparative lower distillation was observed in $W$-shape polythene based solar still though it has $2 \mathrm{~m}^{2}$ areas. It might be due to the use of polythene as a glazing area and more heat loss through the unit. Comparative cost of compact nature of solar stills like single slope, double slope and wick type solar still was more than Rs.7000/- which is four times more than newly developed $W$-shape 3 channel solar still. Comparative output from newly developed solar still was low but it has several advantages that it is cheapest, cost efficient and easy to clean.

Table: 16. Average performance of solar stills in year

\begin{tabular}{|c|c|c|c|c|c|}
\hline $\begin{array}{l}\text { Sr. } \\
\text { No. }\end{array}$ & Type & $\begin{array}{c}\text { distilled } \\
\text { water } \\
\text { (ml/ day) }\end{array}$ & $\begin{array}{c}\text { inside } \\
\text { temperature } \\
\left({ }^{\circ} \mathrm{C}\right)\end{array}$ & $\begin{array}{c}\text { inside } \\
\text { humidity } \\
(\%)\end{array}$ & $\begin{array}{l}\text { App.cost } \\
\text { ( Rs.) }\end{array}$ \\
\hline & Available in marke & & & & \\
\hline 1 & Single slope $\left(1 \mathrm{~m}^{2}\right)$ & 1350 & 60 & 78 & $8000 /-$ \\
\hline 2 & $\begin{array}{l}\text { Double slope }\left(1 \mathrm{~m}^{2}\right) \\
\text { Newly developed }\end{array}$ & 1550 & 70 & 82 & $7500 /-$ \\
\hline 1 & Wick type $\left(1 \mathrm{~m}^{2}\right)$ & 2425 & 83.6 & 95 & $7241 /-$ \\
\hline 2 & $\begin{array}{l}\text { W shape- } 2 \text { channel } \\
\text { on concrete } 2 \mathrm{~m}^{2} \text { ) }\end{array}$ & 1012 & 50.5 & 74.5 & $4000 /-$ \\
\hline 3 & $\begin{array}{l}\text { W shape- } 2 \text { channel } \\
\text { on ground, } 2 \mathrm{~m}^{2}\end{array}$ & 1443 & 53.4 & 75.5 & 1848/- \\
\hline 4 & $\mathrm{~L}$ shape, $\left.2 \mathrm{~m}^{2}\right)$ & 925 & 64.6 & 72 & $1000 /-$ \\
\hline 5 & $\begin{array}{l}\text { W shape- } 3 \text { channel } \\
\text { on ground, } 2 \mathrm{~m}^{2} \text { ) }\end{array}$ & 2104 & 53.5 & 74.9 & 1848/- \\
\hline
\end{tabular}

\section{Chemical Analysis}

Chemical analysis of impure and pure water obtained from $W$-shape three channel solar still was carried for $\mathrm{pH}, \mathrm{EC}$, TDS and ions $\left(\mathrm{Mg}^{++}, \mathrm{Ca}^{++}, \mathrm{Na}^{+}\right.$, 
$\left.\mathrm{CO}_{3}{ }^{-}, \mathrm{HCO}_{3}^{-}, \mathrm{Cl}^{-}\right)$concentration. The concentration of these substrates before and after desalination is given in Table 17.

Table: 17. Chemical analysis of impure and pure water sample

\begin{tabular}{lccc}
\hline $\begin{array}{c}\text { Chemical properties } \\
\text { of Water }\end{array}$ & Tap Water & $\begin{array}{c}\text { Distilled water } \\
\text { obtained from } \\
\text { Millipore unit }\end{array}$ & $\begin{array}{c}\text { Distilled Water } \\
\text { obtained from } \\
\text { solar distillation } \\
\text { unit }\end{array}$ \\
\hline $\mathrm{pH}$ & 7.8 & 7.0 & 7.0 \\
$\mathrm{EC}(\mu \mathrm{S} / \mathrm{cm})$ & 100 & 0.5 & $0.8(0.520 \mathrm{mg} / \mathrm{l})$ \\
$\mathrm{TSS}\left(\%,{ }^{\circ} \mathrm{Brix}\right)$ & 0.02 & 0 & 0 \\
$\mathrm{Mg}^{++}(\mathrm{ppm})$ & 0.00088 & 0 & 0.00036 \\
$\mathrm{Ca}^{++}(\mathrm{ppm})$ & 0.00090 & 0 & 0.00032 \\
$\mathrm{Mg}^{++}+\mathrm{Ca}{ }^{++}(\mathrm{me} / \mathrm{lit})$ & 0.0018 & 0 & 0.00040 \\
$\mathrm{Na}^{+}(\mathrm{me} / \mathrm{lit})$ & 3.5 & 0 & 0.1 \\
$\mathrm{CO}_{3}-(\mathrm{me} /$ lit $)$ & 0.6 & 0 & 0 \\
$\mathrm{HCO}_{3}^{-}(\mathrm{me} / \mathrm{lit})$ & 0.2 & 0 & 0.15 \\
$\mathrm{Cl}^{-}(\mathrm{me} / \mathrm{lit})$ & 1.6 & 0 & 0 \\
\hline
\end{tabular}

It was observed from the results of chemical analysis of pure and impure water, given in Table 7 that there was drastic reduction in the $\mathrm{pH}, \mathrm{EC}, \mathrm{Mg}^{++}$, $\mathrm{Ca}^{++}, \mathrm{Na}^{+}, \mathrm{CO}_{3}^{-}, \mathrm{HCO}_{3}^{-}$ions Carbonate, Bicarbonate etc. in the distilled water.

\section{Cost economics}

During evaluating performance of solar distillation unit, different direct benefits were derived. These benefits were indicators of technical feasibility of plant. Subsequently the economics of the plant was evaluated in the term of cost per liter of distilled water through electrical backup in distillation unit. Hourly benefits of the plant are considered and payback period of distillation unit was made. The total cost that of the investment spread over the entire useful life of the plant, including initial cost, operation cost, maintenance and interest are taken in consideration for payback period.

Considering the average distilled water obtained from even type $W$ shape solar still as 2.1 liter for 250 days a year. It produced 526 liters of distilled 
water yearly. By considering the wholesale market value of distilled water (Rs.10/lit) and total income generated while producing 526 liters of distilled water is tabulated in Table 18.

Table: 18. Details about cost analysis of solar still

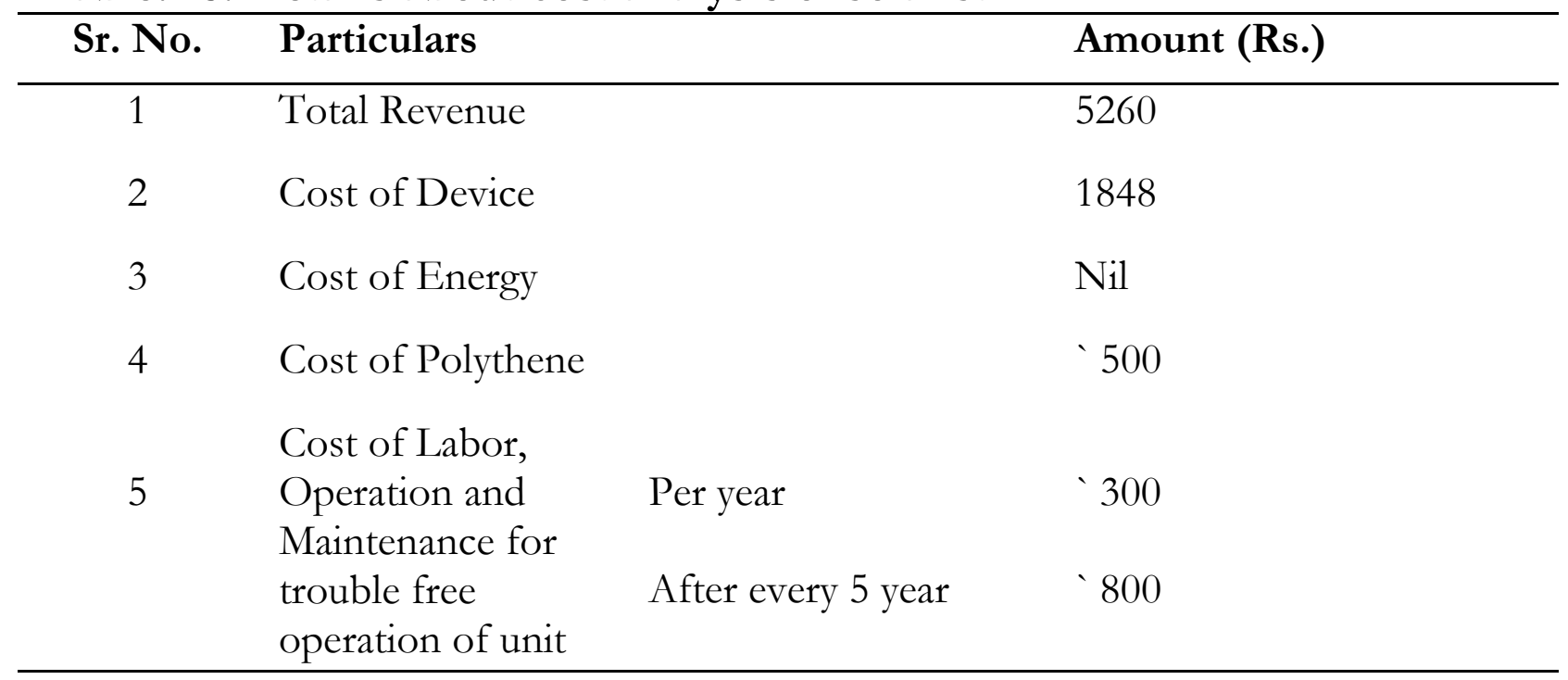

It was observed from the Table 20, the cost of unit is recovered within 4 months 6 days only, i.e. the payback period of the unit was only $1 / 3^{\text {rd }}$ year and after that period the unit will produce net profit. Area of newly developed solar still is double than other two distiller but pay back period is minimum it may due to the lower cost of unit. All economic indicators are summarized in Table 20, as Benefit Cost Ratio (BCR) was 2.56, whereas Net Present Worth (NPW) was Rs.38958. 
Table: 19. Payback Period Analysis of distillation unit

\begin{tabular}{cccccc}
\hline Year & $\begin{array}{c}\text { Cash } \\
\text { outflow }\end{array}$ & $\begin{array}{l}\text { PW of Cash } \\
\text { outflow (at 12 \% } \\
\text { discount rate) }\end{array}$ & $\begin{array}{l}\text { Cash } \\
\text { inflow }\end{array}$ & $\begin{array}{l}\text { PW of Cash } \\
\text { inflow (at 12 \% } \\
\text { discount rate) }\end{array}$ & NPW \\
\hline $\mathbf{A}$ & $\mathbf{B}$ & $\mathbf{C}$ & $\mathbf{D}$ & $\mathbf{F}$ & F-C \\
0 & 1848 & 1848 & 0.0 & & -1848.0 \\
$\mathbf{1}$ & $\mathbf{3 0 0}$ & 270.3 & 5260.0 & 4738.7 & $\mathbf{4 4 6 8 . 5}$ \\
2 & 300 & 243.5 & 5260.0 & 4269.1 & 4025.6 \\
3 & 300 & 219.4 & 5260.0 & 3846.1 & 3626.7 \\
4 & 300 & 197.6 & 5260.0 & 3464.9 & 3267.3 \\
5 & 800 & 474.8 & 5260.0 & 3121.6 & 2646.8 \\
6 & 300 & 160.4 & 5260.0 & 2812.2 & 2651.8 \\
7 & 300 & 144.5 & 5260.0 & 2533.5 & 2389.0 \\
8 & 300 & 130.2 & 5260.0 & 2282.5 & 2152.3 \\
9 & 300 & 117.3 & 5260.0 & 2056.3 & 1939.0 \\
10 & 800 & 281.7 & 5260.0 & 1852.5 & 1570.7 \\
11 & 300 & 95.2 & 5260.0 & 1668.9 & 1573.7 \\
12 & 300 & 85.8 & 5260.0 & 1503.5 & 1417.8 \\
13 & 300 & 77.3 & 5260.0 & 1354.5 & 1277.3 \\
14 & 300 & 69.6 & 5260.0 & 1220.3 & 1150.7 \\
15 & 800 & 167.2 & 5260.0 & 1099.4 & 932.2 \\
16 & 300 & 56.5 & 5260.0 & 990.4 & 933.9 \\
17 & 300 & 50.9 & 5260.0 & 892.3 & 841.4 \\
18 & 300 & 45.8 & 5260.0 & 803.8 & 758.0 \\
19 & 300 & 41.3 & 5260.0 & 724.2 & 682.9 \\
20 & 0 & 0.0 & 5260.0 & 652.4 & 652.4 \\
\hline \multicolumn{7}{|c|}{ TOTAL } & $\mathbf{2 9 2 9 . 1}$ & & 41887.1 & $\mathbf{3 8 9 5 8 . 0}$ \\
\hline
\end{tabular}

Table: 20. Economic indicators for solar distillation units

\begin{tabular}{lcccl}
\hline \multicolumn{1}{c}{ Type } & $\begin{array}{c}\text { Cost } \\
\text { (Rs.) }\end{array}$ & $\begin{array}{c}\text { Net Present } \\
\text { Worth }\end{array}$ & $\begin{array}{c}\text { BCR for } \\
\text { first year }\end{array}$ & PBP \\
\hline $\begin{array}{l}\text { Even type } \\
\text { distillation }\end{array}$ & $1848 /-$ & 38958 & 2.56 & 4 months 6 \\
unit & & & & days \\
\hline
\end{tabular}




\section{Conclusions}

1. Newly developed $W$-shape solar still with three channel erected on soil was economical for the average output of $2104 \mathrm{ml} /$ day distilled water.

2. Concentration of $\mathrm{pH}, \mathrm{EC}$, TDS and ions in solar distilled water was found to be similar as conventional distilled water.

\section{References}

Adhikari, R.S., Kumar, A., Sodha, G.D., 1995. Simulation studies on a multistage stacked tray solar still. J. Solar Energy 54(5), 317.

Ahmed, S.T., 1988. Study of single effect solar still with an internal condenser. Int. J. Solar Wind Tech. 5 (6), 637.

Anna, M. and S. Rangarajan, 1980. Solar Radiation over India, Allied Publisher Privet Ltd., New Delhi : 404

Ashok Kumar and J.D. Anand., 1992. Modeling and performance of a tubular multiwick solar still. Energy-an international journal, 17(11):1067-71.

Aybar, H. S. et al.,2005. An experimental study on an inclined solar water distillation system. Desalination 180, 285-289

Bapeshwar, V., Tiwari, G.N., 1984. Effect of water flow over the glass on the performance of a solar still coupled with a flat plate collector. Int. J. Solar Energy 2, 277.

Barrera, E., 1992. A technical and economical analysis of solar water still in Mexico. J. Renewable Energy 2 (4), 489.

Bassam, A., Hijleh, K.A., Mousa, H.A., 1997. Water film cooling over the glass cover of a solar still including evaporation effects. J. Energy 22, 43.

Bassam, A., Hijleh, K.A., Rababa'h, H.M., 2003. Experimental study of a solar still with sponge cubes in basin. J. Energy Convers. Manag. 44 (9), 1411.

Bhatt, R. N., 1996. Performance evaluation of solar still, unpublished B. TechThesis.1996

Brenidorfer, B. et al., 1995. Solar dryer; their role in post harvest processing, Commonwealth Secretariat Marlborough house, London, Swly 5hx.

Bongirwar, D. R., 1998. Utilization of solar energy in agricultural sector. Industrial products finder

Bouchekima, B., 2003. Solar desalination plant for small size use in remote arid areas of South Algeria for the production of drinking water. Desalination $15 x$. 
Bouchekima, B., 2003. Solar desalination plant for small size use in remote arid areas of South Algeria for the production of drinking water. Desalination 156, 353-354.

Chafik, E., 2003. A new type of seawater desalination plants using solar energy. Desalination 156, 333-348.

Clark, J.A., 1990. The steady-state performance of a solar still. J. Solar Energy 44 (1), 43.

Coffey, J.P., 1975. Vertical solar distillation: technical note. J. Solar Energy 17, 375.

Cooper, P.I., 1969. Digital simulation of transient solar still processes. J. Solar Energy 12 (3), 313.

Cooper, P.I., 1970. The transient analysis of glass covered solar still. Ph.D. Thesis. University of Western Australia, Australia.

Dutt, D. K., A.. Kumar; J. D. Anand, G. N. Tiwari., 1994. Improved design of a double effect solar still. Teri Information and Energy Digest,4(1):55

EI-Swify, M.E. and M.Z. Metias, 2002. Performance of double exposure solar still. Renewable Energy, 26(4):531-547.

Fraca, K. B., 2001. Design and performance of small scale solar powered water desalination system. Teri Information and Energy Digest, 11(2): 118

Gourds, J. and H.Cracken., 1985. Under standing solar stills. Verginia, USA, VITA, 45.

Jigneesh, N. and Rangacharyulum, M., 1989. Development of low cost solar water still for drought-hit areas. Renewable Energy and Enviroment Proceeding of Natural Solar Energy Convention, Udaipur, India, 1-3 December: 271-5.

Ihalawela, P. H. C. A. and Careem, M. A., 2007. A cheap automatic solar water distiller. Proceedings of the technical sessions 23, 41-45.

John, S., et al., 1989. Studies on wick type solar still with removable glass cover. Renewable Energy for Rural Development: Proceeding of the National Solar Energy convention Hydrabad, India 1-3, December: 295-9.

Kothari, S. and Sengar, S.H., 2007. Performance evaluation of solar stills. Bioved, 18(1,2): 35-37

Kumar, S. and G.N. Tiwari, 1996. Performance evolution of an active solar distillation system. Energy-an International Journal,21(9):805-808.

Lawrence, S., Gupta, S., Tiwari, G.N., 1988. Experimental validation of thermal analysis of solar still with dye. Int. J. Solar Energy 6, 291.

Lawrence, S.A., Tiwari, G.N., 1990. Theoretical evaluation of solar distillation under natural circulation with heat exchanger. J. Energy Convers. Manag. 30, 205. 
Lawrence, S.A., Gupta, S.P., Tiwari, G.N., 1990. Effect of heat capacity on the performance of solar still with water flow over the glass cover. J. Energy Convers. Manag. 30, 277.

Lof, G.O.G., Eibling, J.A., Bloemer, J.W., 1961. Energy balances in solar distillation. J. Am. Inst. Chem. Eng. 7 (4), 641.

Malik, M.A.S., Tiwari, G.N., Kumar, A., Sodha, M.S., 1982. Solar Distillation. Pergamon Press, Oxford, UK.

Mouchot, A., 1869. La Chaleur Solavie et ses Applications. Gauthier-Villars, Paris.

Mukherjee, K., Tiwari, G.N., 1986. Economic analysis of various designs of conventional solar stills. J. Energy Convers. Manag. 26, 155.

Murugavel, K.K. et al., 2008. An experimental study on single basin double slope simulation solar still with thin layer of water in the basin. Desalination 220, 687-693

Nayak, J.K., Tiwari, G.N., Sodha, M.S., 1980. Periodic theory of solar still. Int. J. Energy Res. 4, 41.

Nebbia, G., Mennozi, G., 1966. A short history of water desalination, In: Proceedings of International Symposium, Milano, pp. 129.

Patil, C.B. and Patil, V.H., 1989. Weekly Rainfall Probabilities In North Konkan Coastal Zone of Maharashtra State. Research Bulletin Agromet No.5 Regional Agricultural Research Station, Karjat, Konkan Krishi Vidyapeeth, pp-3

Rahman, M. H. et al. 1997. Pilot solar desalination plants in Bangladesh. In: $23^{\text {rd }}$ WEDC conference, p.178-181.

Rai, G. D. 1991. Solar Energy Utillization, Khanna Publications, $4^{\text {th }}$ edition,pp.197

Rai, S.N., Tiwari, G.N., 1982. Single basin solar still coupled with flat plate collector. J. Energy Convers. Manag. 23, 145.

SAFI, M. J., 2004. Solar Ponds For Thermal Desalination And Membrane Distillation, Engineering School of Tunis- TUNISIA, 14(9): 28-54

Singh, P.P. and Mannan, K. D., 1983. Some studies on solar distillation. Prock of National Solar Energy Convention, Hydrabad, India.

Seshadri, C. V., et al., 1995. Design of low cost solar still. Teri Information and Energy Digest, 5(1): 139

Singh, A. K. and Tiwari, G. N., 1992. Experimental validation of passive regenerative solar still. International Journal of Energy Research, 16(6): 497-506 
Sinha, S. and Kumar, S., 1993. Economics evaluation of passive and active solar distillation systems.In N.K. Bansal (eds.):7-12 New Delhi: Tata Mcgrawhill.

Sinha, S., Tiwari, G. N. and Sodha. M. S., 1992. Techno-economic analysis of solar distillation system. International Journal of Energy Reserch.16 (5):365-372.

Spiegler, K.S. and Y.M. El-Sayed. 2001. "The Energetics of Desalination Processes."

Desalination. 134: 109-128.

Srivastava, S. C., 1984. Solar still. Rural Technology Journal, 1 (2-3): 34-40

Tyagi, S. and Rathore, N. S., 2000. Performance evaluation of Solar distillation plants. Commercialization Aspects of Renewable Energy Sources.4648

Thanvi, K.P. and Pande, P. C., 1988. Development of solar still for rose water production: Proceeding of the National Solar Energy Convention, Hydrabad, India, 1-3 December

Thanvi, K.P., 1982. Design and Development of a multibasin tilted type solar still. Allied Publisher, New Delhi.

Thavi, K. P. and Pande, P. C., 1990. Development of inclined solar dryer with alternative materials. Renewable Energy and Environment proceeding of Natural Solar Energy Convention, Udaipur, India, 1-3 December:271-5

Tiris, C. M. Tiris., Y. 1998. Effect of collector Orientation on Solar Energy availability. Teri Information and Energy Digest,9(2)

Tiris, C. M. Tiris, Y. Erdalli and M. Sohmen, 1999. Experimental studies on a solar still coupled with a flat plate collector and a single basin still. Teri Information and Energy Digest,9(3)

Tiwari, G. N. and S. Suneja, 1998. Performance evaluation of an inverted absorber solar still. Energy Conservation and Management: an International Journal, 39 (3/4):173-180.

Tiwari, G.N., S. Sinha and P. Saxena, 1993. Standard Testing procedure for solar distiller unit. RERIC International Energy Journal, 14(1):17-24.

Tiwari, G.N. and Tiwari A.K., 2008. Solar distillation practice for water desalination systems. p.244-248.

Tiwari, G. N. et al., 2003. Present status of solar distillation. Solar Energy 75, 367-373

Vanuatu Renewable Energy and Power Association, Feb- 2006. A Demonstration of Solar Stills to Desalinate Seawater Phase- 2, Project Proposal, pp-17 
Venkatesh, A. and Chaturvedi, A. K., 1989. Experimental observations on a portable solar still. Solar Energy Society of India, 3(1):9-13

Wagh, A., 1991. Solar water heating system for apartments. IREDA News, 10(1):21-22 